\title{
INDUCED REPRESENTATIONS OF THE TWO PARAMETRIC QUANTUM DEFORMATION U ${ }_{p q}[\operatorname{gl}(2 / 2)]$
}

\author{
Nguyen Anh Ky \\ Department of Physics, Chuo University, Kasuga, Bunkyo-ku \\ Tokyo 112-8551, Japan \\ and \\ Institute of Physics, P.O. Box 429, Bo Ho, Hanoi 10000, Vietnam
}

\begin{abstract}
The two-parametric quantum superalgebra $U_{p, q}[g l(2 / 2)]$ and its induced representations are considered. A method for constructing all finite-dimensional irreducible representations of this quantum superalgebra is also described in detail. It turns out that finite-dimensional representations of the two-parametric $U_{p, q}[g l(2 / 2)]$, even at generic deformation parameters, are not simply trivial deformations from those of the classical superalgebra $g l(2 / 2)$, unlike the one-parametric cases.
\end{abstract}

$\underline{\text { Running title }}$ : Representations of $U_{p, q}[g l(2 / 2)]$.

PACS numbers : $\quad 02.20 \mathrm{Tw}, 11.30 \mathrm{~Pb}$.

MSC-class. : 81R50; 17A70. 


\section{Introduction}

Introduced in 80 's as a result of the study on quantum integrable systems and Yang-Baxter equations [1] the quantum groups [2]-[7] have been intensively investigated in different aspects. Since then many (algebraic and geometric) structures and various applications of quantum (super) groups have been found (see in this context, for example, Refs. [8, 9, [10, 11]). It turns out that quantum groups are related to unrelated, at first sight, areas of both physics and mathematics (Refs. [8]-[15] and references therein). For applications of quantum groups, as in the non-deformed cases, we often need their explicit representations. However, despite of remarkable results in this direction the problem of investigating and constructing explicit representations of quantum groups, especially those for quantum superalgebras, is still far from being satisfactorily solved. Even in the case of one-parametric quantum superalgebras, explicit representations are mainly known for quantum Lie superalgebras of lower ranks and of particular types like $U_{q}[\operatorname{osp}(1 / 2)], U_{q}[g l(1 / n)]$ (Refs. [15, 16, 17]), while for higher rank quantum Lie superalgebras [18]-[23], besides some q-oscillator representations which are most popular among those constructed, we do not know so much about other representations, in particular, the finite-dimensional ones which in many cases are related to trigonometric solutions of the quantum Yang-Baxter equations [1, 8]. Some general aspects and the module structure finite-dimensional representations of the quantum superalgebras $U_{q}[g l(m / n)]$ are considered in Ref. [21] but, unfortunately, their explicit construction is still absent. So far explicit finite-dimensional irreducible representations are all known and classified only for 
those $U_{q}[g l(m / n)]$ with both $m$ and $n \leq 2$ (see Refs. [15, 22, 23]).

What about multi-parametric deformations (first considered in [4]), this area is even less covered and results are much poorer. Some kinds of two-parametric deformations have been considered by several authors from different points of view (see Refs. 24, 25] and references therein) but, to our knowledge, explicit representations are known and/or classified in a few lower rank cases such as $U_{p, q}[\operatorname{sl}(2 / 1)]$ and $U_{p, q}[g l(2 / 1)]$ only [25, 26]. The latter two-parametric quantum superalgebra $U_{p, q}[g l(2 / 1)]$ was consistently defined and investigated in [25] where all its finitedimensional irreducible representations were explicitly constructed and classified at generic deformation parameters. This $U_{p, q}[g l(2 / 1)]$, however, is still a small quantum superalgebra which can be defined without the so-called extra-Serre defining relations [27, 28, 29] representing additional constraints on odd Chevalley generators in higher rank cases. Now, in order to include the extra-Serre relations on examination we consider a bigger two-parametric quantum superalgebra, namely $U_{p, q}[g l(2 / 2)]$, and its representations. This quantum superalgebra $U_{p, q}[g l(2 / 2)]$ resembles to the one-parametric quantum superalgebra $U_{\sqrt{p q}}[g l(2 / 2)]$ but can not be identified with the latter. Here we suppose $p \neq q$, otherwise we should return to the case of $U_{q}[g l(2 / 2)]$ investigated already in [22, 23]. Another our motivation is that already in the non-deformed case, the superalgebras $g l(n / n)$, especially, their subalgebras $\operatorname{sl}(n / n)$ and $\operatorname{psl}(n / n)$, have special properties (in comparison with other $g l(m / n), m \neq n)$ and, therefore, attract interest [30]. Additionally, structures of two-parameter deformations considered in [25] and here are, of course, richer than 
those of one-parameter deformations. Every deformation parameter can be independently chosen to take a separate generic value (including zero) or to be a root of unity.

Combining the advantages of the previously developed methods for $U_{q}[g l(2 / 2)]$ and $U_{p, q}[g l(1 / 2)]$ (see Refs. 222, 23, 25]) we can construct all finite-dimensional representations of the two-parametric quantum Lie superalgebra $U_{p, q}[g l(2 / 2)]$. In the frame-work of this paper we consider representations at generic $p$ and $q$ only (i.e., $p$ and $q$ are not roots of unity), while representations at roots of unity are a subject of later separate investigations. In comparison with previous papers [22, 25], the approach here is somewhat modified because of some specific features arising in the present case but the main steps in the construction procedure remain the same. Following this approach we can directly construct explicit representations of the quantum superalgebra $U_{p, q}[g l(2 / 2)]$ induced from some (usually, irreducible) finitedimensional representations of the even subalgebra $U_{p, q}[g l(2) \oplus g l(2)]$ which itself is a quantum algebra. Since the latter is a stability subalgebra of $U_{p, q}[g l(2 / 2)]$ we expect the representations of $U_{p, q}[g l(2 / 2)]$ constructed are decomposed into finitedimensional irreducible representations of $U_{p, q}[g l(2) \oplus g l(2)]$. For a clear description of this decomposition we shall introduce a $U_{p, q}[g l(2 / 2)]$-basis (i.e., a basis within a $U_{p, q}[g l(2 / 2)]$-module or briefly a basis of $\left.U_{p, q}[g l(2 / 2)]\right)$ which will be convenient for us in investigating the module structure. This basis (see (4.26)) can be expressed in terms of some basis of the even subalgebra $U_{p, q}[g l(2) \oplus g l(2)]$ which in turn represents a (tensor) product between two $U_{p, q}[g l(2)]$-bases referred to as the 
left and the right ones. As is shown in [25], the Gel'fand-Zetlin (GZ) patterns can serve again as a basis of finite-dimensional representations of $U_{p, q}[g l(2)]$. Thus, finite-dimensional representations of $U_{p, q}[g l(2) \oplus g l(2)]$ are realized in tensor products of two such $U_{p, q}[g l(2)]$ GZ bases. For generic $p$ and $q$, the finite-dimensional $U_{p, q}[g l(2 / 2)]$-modules constructed have similar structures to those of $U_{q}[g l(2 / 2)]$ investigated in [22, 23] and to those of $g l(2 / 2)$ investigated in [31]. However, finitedimensional representations of $U_{p, q}[g l(2 / 2)]$ at generic deformation parameters are not simply trivial deformations from those of $g l(2 / 2)$ that is the former can not be obtained from the latter by putting quantum deformation brackets in appropriate places, unlike many cases of one-parametric deformations. When one or both of $p$ and $q$ are roots of unity the structures of $U_{p, q}[g l(2 / 2)]$-modules are drastically different but we hope that the present method for construction of finite-dimensional representations of $U_{p, q}[g l(2 / 2)]$ at generic deformation parameters can be extended on its finite-dimensional representations at roots of unity.

This paper is organized as follows. The two-parametric quantum superalgebra $U_{p, q}[g l(2 / 2)]$ is consistently defined in section 2 where we also describe how to construct its representations induced from representations of the stability subalgebra $U_{p, q}[g l(2) \oplus g l(2)]$. Section 3 is devoted to constructing finite-dimensional representations of $U_{p, q}[g l(2 / 2)]$. Finally, some comments and conclusions are made in section 4 .

Let us list some abbreviations and notations used throughout the paper: 
fidirmod(s) : finite-dimensional irreducible module(s),

GZ basis : Gel'fand-Zetlin basis,

QGZ basis : Quasi-Gel'fand-Zetlin basis,

lin.env. $\{\mathrm{X}\}$ : linear envelope of $\mathrm{X}$,

$p, q:$ the deformation parameters,

$[x] \equiv[x]_{p, q}=\frac{q^{x}-p^{-x}}{q-p^{-1}}, \quad:$ a $p q$-deformation of a number or an operator $x$,

$V_{l}^{p, q} \otimes V_{r}^{p, q}:$ a tensor product between two linear spaces $V_{l}^{p, q}$ and $V_{r}^{p, q}$ or a tensor product between a $U_{p, q}\left[g l(2)_{l}\right]$-module $V_{l}^{p, q}$ and a $U_{p, q}\left[g l(2)_{r}\right]$-module $V_{r}^{p, q}$,

$T^{p, q} \odot V_{0}^{p, q}:$ a tensor product between two $U_{p, q}[g l(2) \oplus g l(2)]$-modules $T^{p, q}$ and $V_{0}^{p, q}$,

$[E, F\}$ : supercommutator between $E$ and $F$,

$[E, F]_{r} \equiv E F-r F E$ : an r-deformed commutator between $E$ and $F$,

We hope the notations $[x] \equiv[x]_{p, q}$ for quantum deformations, $[m]$ for highest weights (signatures) in (quasi-) GZ bases $(m)$ and $[$,$] for commutators do not confuse the$ reader.

\section{The quantum superalgebra $U_{p, q}[g l(2 / 2)]$}

The quantum superalgebra $U_{p, q} \equiv U_{p, q}[g l(2 / 2)]$ as a two-parametric deformation 
of the universal enveloping algebra $U[g l(2 / 2)]$ of the Lie superalgebra $g l(2 / 2)$ is generated by the operators $L_{k}, E_{12}, E_{23}, E_{34}, E_{21}, E_{32}, E_{43}$ and $E_{i i}(1 \leq i \leq 4)$ called again Cartan-Chevalley generators and satisfying [32]

a) the super-commutation relations $(1 \leq i, i+1, j, j+1 \leq 4)$ :

$$
\begin{array}{ll}
{\left[E_{i i}, E_{j j}\right]} & =0, \\
{\left[E_{i i}, E_{j, j+1}\right]} & =\left(\delta_{i j}-\delta_{i, j+1}\right) E_{j, j+1}, \\
{\left[E_{i i}, E_{j+1, j}\right]} & =\left(\delta_{i, j+1}-\delta_{i j}\right) E_{j+1, j}, \\
{\left[\text { even generator, } L_{k}\right]} & =0, \quad k=1,2,3, \\
{\left[E_{i, i+1}, E_{j+1, j}\right\}} & =\delta_{i j}\left(\frac{q}{p}\right)^{L_{i}-h_{i}\left(1+\delta_{i 2}\right) / 2}\left[h_{i}\right],
\end{array}
$$

with $h_{i}=\left(E_{i i}-\frac{d_{i+1}}{d_{i}} E_{i+1, i+1}\right), L_{1}=L_{l}, L_{2}=0, L_{3}=L_{r}$ and $d_{1}=d_{2}=-d_{3}=-d_{4}$ $=1$,

b) the Serre-relations:

$$
\begin{aligned}
{\left[E_{12}, E_{34}\right] } & =\left[E_{21}, E_{43}\right]=0 \\
E_{23}^{2} & =E_{32}^{2}=0 \\
{\left[E_{12}, E_{13}\right]_{p}=\left[E_{21}, E_{31}\right]_{q} } & =\left[E_{24}, E_{34}\right]_{q}=\left[E_{42}, E_{43}\right]_{p}=0
\end{aligned}
$$

and

c) the extra-Serre relations:

$$
\begin{aligned}
& \left\{E_{13}, E_{24}\right\}=0, \\
& \left\{E_{31}, E_{42}\right\}=0 .
\end{aligned}
$$


Here, the operators

$$
\begin{aligned}
& E_{13}:=\left[E_{12}, E_{23}\right]_{q^{-1}}, \\
& E_{24}:=\left[E_{23}, E_{34}\right]_{p^{-1}}, \\
& E_{31}:=-\left[E_{21}, E_{32}\right]_{p^{-1}}, \\
& E_{42}:=-\left[E_{32}, E_{43}\right]_{q^{-1}}
\end{aligned}
$$

and the operators composed in the following way

$$
\begin{aligned}
& E_{14}:=\left[E_{12},\left[E_{23}, E_{34}\right]_{p^{-1}}\right]_{q^{-1}} \equiv\left[E_{12}, E_{24}\right]_{q^{-1}}, \\
& E_{41}:=\left[E_{21},\left[E_{32}, E_{43}\right]_{q^{-1}}\right]_{p^{-1}} \equiv-\left[E_{21}, E_{42}\right]_{p^{-1}}
\end{aligned}
$$

are defined as new generators which, like $E_{23}$ and $E_{32}$, are all odd and have vanishing squares. These generators $E_{i j}, 1 \leq i, j \leq 4$, are two-parametric deformation analogues ( $p q$-analogues) of the Weyl generators $e_{i j}, 1 \leq i, j \leq 4$, of the superalgebra $g l(2 / 2)$ whose universal enveloping algebra $U[g l(2 / 2)]$ is a classical limit of $U_{p, q}[g l(2 / 2)]$ when $p, q \rightarrow 1$. The so-called maximal-spin operators $L_{k}$ are constants within a $U_{p, q}[g l(2)]$-fidirmod and are different for different $U_{p, q}[g l(2)]$-fidirmods. Therefore, commutators between these operators with the odd generators intertwining $U_{p, q}[g l(2)]$-fidirmods take concrete forms on concrete basis vectors. Other commutation relations between $E_{i j}$ follow from the relations (2.1)-(2.3) and the definitions (2.4) and (2.5). 
The subalgebra $U_{p, q}\left[g l(2 / 2)_{0}\right]\left(\subset U_{p, q}[g l(2 / 2)]_{0} \subset U_{p, q}[g l(2 / 2)]\right)$ is even and isomorphic to $U_{p, q}[g l(2) \oplus g l(2)] \equiv U_{p, q}[g l(2)] \oplus U_{p, q}[g l(2)]$ which is completely defined by $L_{1}, L_{3}, E_{12}, E_{34}, E_{21}, E_{43}$ and $E_{i i}, 1 \leq i \leq 4$,

$$
U_{q}\left[g l(2 / 2)_{0}\right]=\text { lin.env. }\left\{L_{1}, L_{3}, E_{i j} \| i, j=1,2 \text { and } i, j=3,4\right\} .
$$

In order to distinguish two components $U_{p, q}[g l(2)]$ of $U_{p, q}\left[g l(2 / 2)_{0}\right]$ we set

$$
\begin{aligned}
& \text { left } U_{p, q}[g l(2)] \equiv U_{p, q}\left[g l(2)_{l}\right]:=\text { lin.env. }\left\{L_{1}, E_{i j} \| i, j=1,2\right\}, \\
& \text { right } U_{p, q}[g l(2)] \equiv U_{p, q}\left[g l(2)_{r}\right]:=\text { lin.env. }\left\{L_{3}, E_{i j} \| i, j=3,4\right\},
\end{aligned}
$$

that is

$$
U_{p, q}\left[g l(2 / 2)_{0}\right]=U_{p, q}\left[g l(2)_{l} \oplus g l(2)_{r}\right]
$$

Looking at the relations (2.1)-(2.3) we see that every of the odd spaces $A_{+}$ and $A_{-}$spanned on the positive and negative odd roots (generators) $E_{i j}$ and $E_{j i}$, $1 \leq i \leq 2<j \leq 4$, respectively

$$
\begin{aligned}
& A_{+}=\text {lin.env } .\left\{E_{14}, E_{13}, E_{24}, E_{23}\right\}, \\
& A_{-}=\text {lin.env. }\left\{E_{41}, E_{31}, E_{42}, E_{32}\right\},
\end{aligned}
$$

is a representation space of the even subalgebra $U_{p, q}\left[g l(2 / 2)_{0}\right]$ which, as seen from (2.1)-(2.2), is a stability subalgebra of $U_{p, q}[g l(2 / 2)]$. Therefore, we can construct representations of $U_{p, q}[g l(2 / 2)]$ induced from some (finite-dimensional irreducible, for example) representations of $U_{p, q}\left[g l(2 / 2)_{0}\right]$ which are realized in some representation spaces (modules) $V_{0}^{p, q}$ representing tensor products of $U_{p, q}\left[g l(2)_{l}\right]$-modules $V_{0, l}^{p, q}$ and $U_{p, q}\left[g l(2)_{r}\right]-$ modules $V_{0, r}^{p, q}$

$$
V_{0}^{p, q}(\Lambda)=V_{0, l}^{p, q}\left(\Lambda_{l}\right) \otimes V_{0, r}^{p, q}\left(\Lambda_{r}\right)
$$


where $\Lambda$ 's are some signatures (such as highest weights, respectively) characterizing the modules (highest weight modules, respectively). Here $\Lambda_{l}$ and $\Lambda_{r}$ are referred to as the left and the right components of $\Lambda$, respectively

$$
\Lambda=\left[\Lambda_{l}, \Lambda_{r}\right]
$$

If we demand

$$
E_{23} V_{0}^{p, q}(\Lambda)=0
$$

hence

$$
U_{p, q}\left(A_{+}\right) V_{0}^{p, q}=0
$$

we turn the $U_{p, q}\left[g l(2 / 2)_{0}\right]$-module $V_{0}^{p, q}$ into a $U_{p, q}(B)$-module where

$$
B=A_{+} \oplus g l(2) \oplus g l(2)
$$

The $U_{p, q}[g l(2 / 2)]-$ module $W^{p, q}$ induced from the $U_{p, q}\left[g l(2 / 2)_{0}\right]-$ module $V_{0}^{p, q}$ is the factor-space

$$
W^{p, q}=W^{p, q}(\Lambda)=\left[U_{p, q} \otimes V_{0}^{p, q}(\Lambda)\right] / I^{p, q}(\Lambda)
$$

which, of course, depends on $\Lambda$, where

$$
U_{p, q} \equiv U_{p, q}[g l(2 / 2)]
$$

while $I^{p, q}$ is the subspace

$$
I^{p, q}=\text { lin.env. }\left\{u b \otimes v-u \otimes b v \| u \in U_{p, q}, b \in U_{p, q}(B) \subset U_{p, q}, v \in V_{0}^{p, q}\right\} .
$$

Using the commutation relations (2.1)-(2.3) and the definitions (2.4) and (2.5) we can prove the following analogue of the Poincaré-Birkhoff-Witt theorem 
Proposition 1: The quantum deformation $U_{p, q}:=U_{p, q}[g l(2 / 2)]$ is spanned on all possible linear combinations of the elements

$$
g=\left(E_{23}\right)^{\eta_{1}}\left(E_{24}\right)^{\eta_{2}}\left(E_{13}\right)^{\eta_{3}}\left(E_{14}\right)^{\eta_{4}}\left(E_{41}\right)^{\theta_{1}}\left(E_{31}\right)^{\theta_{2}}\left(E_{42}\right)^{\theta_{3}}\left(E_{32}\right)^{\theta_{4}} g_{0}
$$

or equivalently

$$
g=\left(E_{41}\right)^{\theta_{1}}\left(E_{31}\right)^{\theta_{2}}\left(E_{42}\right)^{\theta_{3}}\left(E_{32}\right)^{\theta_{4}} b
$$

where $g_{0} \in U_{p, q}\left[g l(2 / 2)_{0}\right], b \in U_{p, q}(B)$ and $\eta_{i}, \theta_{i}=0,1$.

Any vector $w$ from the module $W^{p, q}$ can be represented as

$$
w=u \otimes v, \quad u \in U_{p, q}, \quad v \in V_{0}^{p, q} .
$$

Then $W^{p, q}$ is a $U_{p, q}[g l(2 / 2)]-$ module in the sense

$$
g w \equiv g(u \otimes v)=g u \otimes v \in W^{p, q}
$$

for $g, u \in U_{p, q}, w \in W^{p, q}$ and $v \in V_{0}^{p, q}$. Taking into account the fact that $V_{0}^{p, q}(\Lambda)$ is a $U_{p, q}(B)$-module we have

$$
W^{q}(\Lambda)=\text { lin.env. }\left\{\left(E_{41}\right)^{\theta_{1}}\left(E_{31}\right)^{\theta_{2}}\left(E_{42}\right)^{\theta_{3}}\left(E_{32}\right)^{\theta_{4}} \otimes v \| v \in V_{0}^{p, q}, \theta_{1}, \ldots, \theta_{4}=0,1\right\} \text {. }
$$

Consequently, a basis of $W^{p, q}$ can be constituted by taking all the vectors of the form

$$
\left|\theta_{1}, \theta_{2}, \theta_{3}, \theta_{4} ;(\lambda)\right\rangle:=\left(E_{41}\right)^{\theta_{1}}\left(E_{31}\right)^{\theta_{2}}\left(E_{42}\right)^{\theta_{3}}\left(E_{32}\right)^{\theta_{4}} \otimes(\lambda), \quad \theta_{i}=0,1,
$$

where $(\lambda)$ is a (GZ, for example) basis of $V_{0}^{p, q} \equiv V_{0}^{p, q}(\Lambda)$. We refer to this basis of $W^{p, q}$ as the induced $U_{p, q}[g l(2 / 2)]$-basis (or simply, the induced basis) in order 
to distinguish it from another $U_{p, q}[g l(2 / 2)]$-basis introduced later and called a reduced basis which is more convenient for investigating the module structure of $W^{p, q}$. It is obvious that if the module $V_{0}^{p, q}$ is finite-dimensional so is the module $W^{p, q}$. Finite-dimensional representations of $U_{p, q}[g l(2 / 2)]$ are namely the subject of the next section.

\section{Finite-dimensional representations of $U_{p, q}[g l(2 / 2)]$}

In this section we consider finite-dimensional representations of $U_{p, q}[g l(2 / 2)]$ induced from irreducible finite-dimensional representations of $U_{p, q}\left[g l(2 / 2)_{0}\right]$. We firstly construct the bases of the module $W^{q}$ and then find the explicit matrix elements for the finite-dimensional representations of $U_{p, q}[g l(2 / 2)]$.

We can shown that the GZ patterns

$$
\left[\begin{array}{cc}
m_{12} & m_{22} \\
m_{11}
\end{array}\right] \equiv\left[\begin{array}{l}
{[m]} \\
m_{11}
\end{array}\right]
$$

where $m_{i j}$ are complex numbers such that $m_{12}-m_{11} \in \mathbf{Z}_{+}$and $m_{11}-m_{22} \in \mathbf{Z}_{+}$, can serve as a basis of a $U_{p, q}[g l(2)]$-fidirmod. Indeed, finite-dimesional representations of $U_{p, q}[g l(2)]$ are high weight and if the operators $L$ and $E_{i j}, i, j=1,2$, are difined on the basis (3.1) as follows

$$
L\left[\begin{array}{cc}
m_{12} & m_{22} \\
m_{11}
\end{array}\right]=\frac{1}{2}\left(l_{12}-l_{22}-1\right)\left[\begin{array}{cc}
m_{12} & m_{22} \\
& m_{11}
\end{array}\right],
$$




$$
\begin{aligned}
& E_{11}\left[\begin{array}{ll}
m_{12} & m_{22} \\
m_{11}
\end{array}\right]=\left(l_{11}+1\right)\left[\begin{array}{ll}
m_{12} & m_{22} \\
m_{11}
\end{array}\right], \\
& E_{22}\left[\begin{array}{ll}
m_{12} & m_{22} \\
m_{11}
\end{array}\right]=\left(l_{12}+l_{22}-l_{11}+2\right)\left[\begin{array}{ll}
m_{12} & m_{22} \\
m_{11}
\end{array}\right], \\
& E_{12}\left[\begin{array}{cc}
m_{12} & m_{22} \\
m_{11}
\end{array}\right]=\left(\left[l_{12}-l_{11}\right]\left[l_{11}-l_{22}\right]\right)^{1 / 2}\left[\begin{array}{ll}
m_{12} & m_{22} \\
m_{11}+1
\end{array}\right], \\
& E_{21}\left[\begin{array}{cc}
m_{12} & m_{22} \\
m_{11}
\end{array}\right]=\left(\left[l_{12}-l_{11}+1\right]\left[l_{11}-l_{22}-1\right]\right)^{1 / 2}\left[\begin{array}{ll}
m_{12} & m_{22} \\
m_{11}-1
\end{array}\right],
\end{aligned}
$$

they really satisfy commutation relations of $U_{p, q}[g l(2)]$ given in (2.1). Here the notation

$$
l_{i j}=m_{i j}-i, \quad i=1,2
$$

and later also the notation

$$
l_{i j}^{\prime}=m_{i j}-i+2, \quad i=3,4
$$

are used. Since the $U_{p, q}\left[g l(2 / 2)_{0}\right]$-fidirmod $V_{0}^{p, q}$ is decomposed into a $U_{p, q}\left[g l(2)_{l}\right]_{-}$ fidirmod $V_{0, l}^{p, q}$ and a $U_{p, q}\left[g l(2)_{r}\right]$-fidirmod $V_{0, r}^{p, q}$ via the tensor product

$$
V_{0}^{p, q}=V_{0, l}^{p, q} \otimes V_{0, r}^{p, q},
$$

its basis, therefore, is a tensor product

$$
\left[\begin{array}{cc}
m_{13} & m_{23} \\
m_{11}
\end{array}\right] \otimes\left[\begin{array}{cc}
m_{33} & m_{43} \\
m_{31}
\end{array}\right] \equiv\left[\begin{array}{l}
{[m]_{l}} \\
m_{11}
\end{array}\right] \otimes\left[\begin{array}{c}
{[m]_{r}} \\
m_{31}
\end{array}\right] \equiv(m)_{l} \otimes(m)_{r} \equiv(m)
$$


between a GZ basis of $V_{0, l}^{p, q}$ spanned on the vectors $(m)_{l}$ and a GZ basis of $V_{0, r}^{p, q}$ spanned on the vectors $(m)_{r}$. Following the approach of Ref. [22] (see aslo Ref. 23, 31]) and keeping the notations used there, we can represent the basis (3.5) of $V_{0}^{p, q}$ in the form

$$
\left[\begin{array}{cccc}
m_{13} & m_{23} & m_{33} & m_{43} \\
& & ; & \\
m_{11} & & m_{31}
\end{array}\right] \equiv\left[\begin{array}{ccc}
{[m]_{l}} & {[m]_{r}} \\
& ; & \\
m_{11} & & m_{31}
\end{array}\right] \equiv(m)
$$

Then, the signature $\Lambda$, which now is the highest weight, is given by the first row $\left[m_{13}, m_{23}, m_{33}, m_{43}\right] \equiv\left[[m]_{l},[m]_{r}\right] \equiv[m]$ common for all the basis vectors $(3.6)$ of $V_{0}^{p, q}:$

$$
V_{0}^{p, q} \equiv V_{0}^{p, q}(\Lambda)=V_{0}^{p, q}([m])=V_{0, l}^{p, q}\left([m]_{l}\right) \otimes V_{0, r}^{p, q}\left([m]_{r}\right)
$$

The explicit action of $U_{p, q}\left[g l(2 / 2)_{0}\right]$ on $V_{0}^{p, q}([m])$ follows directly from (3.2) and :

$$
g_{0}(m)=g_{0, l}(m)_{l} \otimes(m)_{r}+(m)_{l} \otimes g_{0, r}(m)_{r}
$$

for $g_{0} \equiv g_{0, l} \oplus g_{0, r} \in U_{q}\left[g l(2 / 2)_{0}\right]$ and $(m) \in V_{0}^{q}([m])$.

The basis vector with $m_{11}=m_{13}$ and $m_{31}=m_{33}$

$$
\left[\begin{array}{cccc}
m_{13} & m_{23} & m_{33} & m_{43} \\
& & ; & \\
m_{13} & & m_{33}
\end{array}\right] \equiv\left[\begin{array}{ccc}
{[m]_{l}} & {[m]_{r}} \\
m_{13} & m_{33}
\end{array}\right] \equiv(M)
$$

satisfying the conditions

$$
\begin{aligned}
& E_{i i}(M)=m_{i 3}(M), \quad i=1,2,3,4, \\
& E_{12}(M)=E_{34}(M)=0
\end{aligned}
$$


is the highest weight vector in $V_{0}^{p, q}([m])$. Therefore, as in the classical case $(p=$ $q=1)$ [31] and in the case of one-parametric deformation $(p=q)$ [22] the highest weight $[m]$ is nothing but an ordered set of the eigen values of the Cartan generators $E_{i i}$ on the highest weight vector $(M)$. The latter is also highest weight vector in $W^{p, q}([m])$ because of the condition (2.14). All other, i.e. lower weight, basis vectors of $V_{0}^{p, q}$ can be obtained from the highest weight vector $(M)$ through acting on the latter by monomials of the lowering generators $E_{21}$ and $E_{43}$ in definite powers:

$$
\begin{aligned}
(m)= & \left(\frac{\left[m_{11}-m_{23}\right] !\left[m_{31}-m_{43}\right] !}{\left[m_{13}-m_{23}\right] !\left[m_{13}-m_{11}\right] !\left[m_{33}-m_{43}\right] !\left[m_{33}-m_{31}\right] !}\right)^{1 / 2} \\
& \times\left(E_{21}\right)^{m_{13}-m_{11}}\left(E_{43}\right)^{m_{33}-m_{31}}(M)
\end{aligned}
$$

where $[n]$ 's stand for

$$
\frac{q^{n}-p^{-n}}{q-p^{-1}} \equiv[n]_{p, q} \equiv[n]
$$

while

$$
[n] !=[1][2] \ldots[n-1][n] .
$$

Therefore, the induced basis $(2.25)$ of $W^{p, q}(\Lambda)=W^{p, q}([m])$ now takes the form

$$
\left|\theta_{1} \theta_{2}, \theta_{3}, \theta_{4} ;(m)\right\rangle:=\left(E_{41}\right)^{\theta_{1}}\left(E_{31}\right)^{\theta_{2}}\left(E_{42}\right)^{\theta_{3}}\left(E_{32}\right)^{\theta_{4}} \otimes(m)
$$

The subspace $T^{p, q}$ consisting of

$$
\left|\theta_{1}, \theta_{2}, \theta_{3}, \theta_{4}\right\rangle:=\left(E_{41}\right)^{\theta_{1}}\left(E_{31}\right)^{\theta_{2}}\left(E_{42}\right)^{\theta_{3}}\left(E_{32}\right)^{\theta_{4}}
$$

can be considered as a $U_{p, q}\left[g l(2 / 2)_{0}\right]$-adjoint module which is 16 -dimensional when all the $\theta_{i}(i=1,2,3,4)$ take all two possible values 0 and 1 , that is $\sum_{i=1}^{4} \theta_{i}$ runs 
all over the range from 0 to 4 . Thus $W^{p, q}([m])$ being a tensor product between two $U_{p, q}\left[g l(2 / 2)_{0}\right]-$ modules:

$$
W^{p, q}([m])=T^{p, q} \odot V_{0}^{p, q}([m])
$$

is, in general, a reducible $U_{p, q}\left[g l(2 / 2)_{0}\right]$-module and are decomposed into irreducible $U_{p, q}\left[g l(2 / 2)_{0}\right]$-submodules. We arrive at the next assertion

Proposition 2: The induced $U_{p, q}[g l(2 / 2)]-$ module $W^{p, q}$ is the linear span

$$
W^{p, q}([m])=\text { lin.env. }\left\{\left(E_{41}\right)^{\theta_{1}}\left(E_{31}\right)^{\theta_{2}}\left(E_{42}\right)^{\theta_{3}}\left(E_{32}\right)^{\theta_{4}} \otimes v \| v \in V_{0}^{p, q}([m]), \theta_{i}=0,1\right\},
$$

which is decomposed into a direct sum of (sixteen, at most) $U_{p, q}\left[g l(2 / 2)_{0}\right]$-fidirmods $V_{k}^{p, q}\left([m]_{k}\right):$

$$
W^{p, q}([m])=\bigoplus_{k=0}^{15} V_{k}^{p, q}\left([m]_{k}\right) .
$$

where $[m]_{k}$ are signatures of $V_{k}^{p, q} \equiv V_{k}^{p, q}\left([m]_{k}\right)$.

Here, we call $[m]_{k} \equiv\left[m_{12}, m_{22}, m_{32}, m_{42}\right]_{k}$ the local highest weights of the submodules $V_{k}^{p, q}$ in their GZ bases denoted now as

$$
\left[\begin{array}{cccc}
m_{12} & m_{22} & m_{32} & m_{42} \\
& ; & \\
m_{11} & & m_{31}
\end{array}\right]_{k} \equiv(m)_{k} .
$$

The highest weight $[m]_{0} \equiv[m]$ of $V_{0}^{p, q}$ being also the highest weight of $W^{p, q}$ is referred to as the global highest weight. We call $[m]_{k}, k \neq 0$, the local highest weights in the sense that they characterize the submodules $V_{k}^{p, q} \subset W^{p, q}$ as $U_{p, q}\left[g l(2 / 2)_{0}\right]$-fidirmods only, while the global highest weight $[m]$ characterizes the $U_{p, q}[g l(2 / 2)]-$ module $W^{p, q}$ 
as the whole. In the same way we define the local highest weight vectors $(M)_{k}$ in $V_{k}^{p, q}$ as those $(m)_{k}$ satisfying the conditions (cf. (3.10))

$$
\begin{aligned}
E_{i i}(M)_{k} & =m_{i 2}(M)_{k}, \quad i=1,2,3,4, \\
E_{12}(M)_{k} & =E_{34}(M)_{k}=0 .
\end{aligned}
$$

The highest weight vector $(M)$ of $V_{0}^{p, q}$ is also the global highest weight vector in $W^{p, q}$ for which the condition (see (2.14))

$$
E_{23}(M)=0
$$

and the conditions (3.18) simultaneously hold.

Let us denote by $\Gamma_{k}^{p, q}$ the basis system spanned on the basis vectors $(m)_{k}(3.17)$ in each $V_{k}^{p, q}([m])$. For a basis of $W^{p, q}$ we can choose the union $\Gamma^{p, q}=\bigcup_{k=0}^{15} \Gamma_{k}^{p, q}$ of all the bases $\Gamma_{k}^{p, q}$, namely, a basis vector of $W^{p, q}$ has to be identified with one of the vectors $(m)_{k}, 0 \leq k \leq 15$. The basis $\Gamma^{p, q}$ is referred to as the $U_{p, q}[g l(2 / 2)]-$ reduced basis or simply, the reduced basis. It is clear that every basis $\Gamma_{k}^{p, q}=\Gamma_{k}\left([m]_{k}\right)^{p, q}$ is labelled by a local highest weight $[m]_{k}$, while the basis $\Gamma^{p, q}=\Gamma^{p, q}([m])$ is labelled by the global highest weight $[m]$. Going ahead, we modify the notation (3.17) for the basis vectors in $\Gamma^{p, q}$ as follows (cf. (4.26) in Ref. [22])

$$
\left[\begin{array}{cccc}
m_{13} & m_{23} & m_{33} & m_{43} \\
m_{12} & m_{22} & m_{32} & m_{42} \\
m_{11} & 0 & m_{31} & 0
\end{array}\right]_{k} \equiv\left[\begin{array}{cccc}
m_{12} & m_{22} & m_{32} & m_{42} \\
& ; & m_{11}
\end{array}\right]_{k} \equiv(m)_{k}
$$

with $k$ running from 0 to 15 as for $k=0$ we must take into account $m_{i 2}=m_{i 3}$, 
$i=1,2,3,4$, i.e.,

$$
(m)_{0} \equiv(m)=\left[\begin{array}{cccc}
m_{13} & m_{23} & m_{33} & m_{43} \\
m_{13} & m_{23} & m_{33} & m_{43} \\
m_{11} & 0 & m_{31} & 0
\end{array}\right]
$$

In (3.20) the first row $[m]=\left[m_{13}, m_{23}, m_{33}, m_{43}\right]$ being the (global) highest weight of $W^{p, q}$ is fixed for all the vectors in the whole $W^{p, q}$ and characterizes this module itself, while the second row is a (local) highest weight of some submodule $V_{k}^{p, q}$ and tells us that the considered basis vector $(m)_{k}$ of $W^{p, q}$ belongs to this submodule in the decomposition (3.16) corresponding to the branching rule $U_{p, q}[g l(2 / 2)] \supset$ $U_{p, q}\left[g l(2 / 2)_{0}\right] \supset U_{p, q}[g l(1) \otimes g l(1)]$. We refer to (3.20) as the quasi-Gel'fand-Zetlin (QGZ) basis.

It is easy to see that the highest vectors $(M)_{k}$ in the notation (3.20) are

$$
(M)_{k}=\left[\begin{array}{cccc}
m_{13} & m_{23} & m_{33} & m_{43} \\
m_{12} & m_{22} & m_{32} & m_{42} \\
m_{12} & 0 & m_{32} & 0
\end{array}\right]_{k}, \quad k=0,1, \ldots 15 .
$$

The (global) highest weight vector $(M)(3.9)$ is given now by

$$
(M)=\left[\begin{array}{cccc}
m_{13} & m_{23} & m_{33} & m_{43} \\
m_{13} & m_{23} & m_{33} & m_{43} \\
m_{13} & 0 & m_{33} & 0
\end{array}\right]
$$

A highest weight vector $(M)_{k}$ expressed in terms of the induced basis (3.14) has the form of a homogeneous polynomial of a definite degree $\eta$ in negative odd generators 
$\left(E_{i j}, 1 \leq j \leq 2<i \leq 4\right)$ acting on $(m) \in V_{0}^{p, q}([m])$ :

$$
(M)_{k} \equiv(M)_{\eta, h}=\sum_{\theta_{i}=0,1} C_{\eta, h}\left(\theta_{1}, \theta_{2}, \theta_{3}, \theta_{4}\right)\left|\theta_{1}, \theta_{2}, \theta_{3}, \theta_{4} ;(m)\right\rangle
$$

with $\eta=\sum_{i=1}^{4} \theta_{i}$ fixed for every $(M)_{\eta, h}$, and the coefficients $C_{\eta, h}$ determined by solving Eqs. (3.18). Applying (3.11) to any $(M)_{\eta, h}$ we find all the basis vectors $(m)_{\eta, h}$ of the corresponding fidirmod $V_{\eta, h}^{p, q}$ which is a linear space spanned on homogeneous polynomials of the negative odd generators of the same degree $\eta$ since (3.11) does not change $\eta$. Here we call $\eta$ the level of $V_{\eta, h}^{p, q}$. It is easy to see that on the level $\eta=0$ there is only one fidirmod, namely $V_{0}^{p, q} \equiv V_{0,1}^{p, q}$, while on the next level $\eta=1$ there are four fidirmods, say, $V_{1, h}^{p, q}, h=1,2,3,4$. On the level $\eta=2$ we can find six fidirmods $V_{2, h}^{p, q}, 1 \leq h \leq 6$, which are divided into two groups $(h=1,2,3$ and $h=$ $4,5,6)$ expressed in terms of two indenpendent groups of second order monomials of odd generators (3.15) acting on $(m)$. For $\eta=3$ the number of fidirmods is four, $V_{3, h}^{p, q}, \quad h=1,2,3,4$, and finally on $\eta=4$ we find again only one fidirmod $V_{4,1}^{p, q}$. However, this form (3.24) which was used in the one-parametric case [22] is now inconvenient for us here to apply formula (3.11) in order to find all other (i.e., lower weight) reduced basis vectors. It is so because of the presence of the maximal-spin operators $L_{i}$ which are not diagonalized in the induced basis but in the reduced basis (since an eigenvalue of any $L_{i}$ is a fixed constant only within a $U_{p, q}[g l(2)]$-fidirmod (or fidirmod, for short) and changes from fidirmod to fidirmod). Applying (3.11) we have to push generators $E_{21}$ and $E_{43}$ to the right side until reaching $V_{0}^{p, q}$ by using commutation ralations $(2.1)-(2.2)$ which give rise to $L_{i}$ 's acting on the induced basis vectors. But it is extremtly difficult to get explicit actions of $L_{i}$ on the latter vectors 
before knowing how they are projected on the reduced basis which, however, we are now looking for. Instead, we will write down $(M)_{k} \equiv(M)_{\eta, h}$ in a form convenient for applying (3.11) which leaves the $\eta$ 's unchanged:

$$
\begin{aligned}
& (M)_{0} \equiv(M)_{0,1}=a_{0}|0,0,0,0 ;(M)\rangle \equiv(M), \quad a_{0} \equiv 1, \\
& (M)_{1} \equiv(M)_{1,1}=a_{1}|0,0,0,1 ;(M)\rangle \equiv a_{1} E_{32}(M), \\
& (M)_{2} \equiv(M)_{1,2}=a_{2}\left\{\frac{1}{a_{1}} E_{21}(M)_{1}-\frac{[2 l+1]}{[2 l]} E_{32} E_{21}(M)\right\}, \\
& (M)_{3} \equiv(M)_{1,3}=a_{3}\left\{\frac{1}{a_{1}} E_{43}(M)_{1}-\frac{\left[2 l^{\prime}+1\right]}{\left[2 l^{\prime}\right]} E_{32} E_{43}(M)\right\}, \\
& (M)_{4} \equiv(M)_{1,4}=a_{4}\left\{\frac{1}{a_{1}} E_{21} E_{43}(M)_{1}-\frac{1}{a_{2}} E_{43}(M)_{2}-\frac{1}{a_{3}} E_{21}(M)_{3}\right. \\
& \left.-\frac{[2 l+1]\left[2 l^{\prime}+1\right]}{[2 l]\left[2 l^{\prime}\right]} E_{32} E_{21} E_{43}(M)\right\} \\
& (M)_{5} \equiv(M)_{2,1}=a_{5}|0,0,1,1 ;(M)\rangle \equiv a_{5} E_{42} E_{32}(M), \\
& (M)_{6} \equiv(M)_{2,2}=a_{6}\left\{\frac{1}{a_{5}} E_{21}(M)_{5}-\frac{[2 l+2]}{[2 l]} E_{42} E_{32} E_{21}(M)\right\}, \\
& (M)_{7} \equiv(M)_{2,3}=a_{7}\left\{\frac{1}{a_{5}} E_{21}^{2}(M)_{5}-\frac{1}{a_{6}} \frac{[2][2 l+1]}{[2 l]} E_{21}(M)_{6}\right. \\
& \left.-\frac{[2 l+1][2 l+2]}{[2 l][2 l-1]} E_{42} E_{32} E_{21}^{2}(M)\right\}, \\
& (M)_{8} \equiv(M)_{2,4}=a_{8}|0,1,0,1 ;(M)\rangle \equiv a_{8} E_{31} E_{32}(M), \\
& (M)_{9} \equiv(M)_{2,5}=a_{9}\left\{\frac{1}{a_{8}} E_{43}(M)_{8}-\frac{\left[2 l^{\prime}+2\right]}{\left[2 l^{\prime}\right]} E_{31} E_{32} E_{43}(M)\right\}, \\
& (M)_{10} \equiv(M)_{2,6}=a_{10}\left\{\frac{1}{a_{8}} E_{43}^{2}(M)_{8}-\frac{1}{a_{9}} \frac{[2]\left[2 l^{\prime}+1\right]}{\left[2 l^{\prime}\right]} E_{43}(M)_{9}\right. \\
& \left.-\frac{\left[2 l^{\prime}+1\right]\left[2 l^{\prime}+2\right]}{\left[2 l^{\prime}\right]\left[2 l^{\prime}-1\right]} E_{31} E_{32} E_{43}^{2}(M)\right\}, \\
& (M)_{11} \equiv(M)_{3,1}=a_{11}|0,1,1,1 ;(M)\rangle \equiv a_{11} E_{31} E_{42} E_{32}(M),
\end{aligned}
$$




$$
\begin{aligned}
(M)_{12} \equiv & (M)_{3,2}=a_{12}\left\{\frac{1}{a_{11}} E_{21}(M)_{11}-\frac{[2 l+1]}{[2 l]} E_{31} E_{42} E_{32} E_{21}(M)\right\}, \\
(M)_{13} \equiv & (M)_{3,3}=a_{13}\left\{\frac{1}{a_{11}} E_{43}(M)_{11}-\frac{\left[2 l^{\prime}+1\right]}{\left[2 l^{\prime}\right]} E_{31} E_{42} E_{32} E_{43}(M)\right\}, \\
(M)_{14} \equiv & (M)_{3,4}=a_{14}\left\{\frac{1}{a_{11}} E_{21} E_{43}(M)_{11}-\frac{1}{a_{12}} E_{43}(M)_{12}-\frac{1}{a_{13}} E_{21}(M)_{13}\right. \\
& \left.-\frac{[2 l+1]\left[2 l^{\prime}+1\right]}{[2 l]\left[2 l^{\prime}\right]} E_{31} E_{42} E_{32} E_{21} E_{43}(M)\right\}, \\
(M)_{15} \equiv & (M)_{4,1}=a_{15}|1,1,1,1 ;(M)\rangle \equiv a_{15} E_{41} E_{31} E_{42} E_{32}(M),
\end{aligned}
$$

where $l=\frac{1}{2}\left(m_{13}-m_{23}\right)$ and $l^{\prime}=\frac{1}{2}\left(m_{33}-m_{43}\right)$, while $a_{k}=a_{k}(p, q)$ are coefficients depending, in general, on $p$ and $q$. Indeed, $(M)_{k}$ given in (3.25) form a set of all linear independent vectors satisfying the conditions (3.18). Looking at (3.25) we easily identify the highest weights $[\mathrm{m}]_{k}$

$$
\begin{aligned}
& {[m]_{0}=\left[m_{13}, m_{23}, m_{33}, m_{43}\right]} \\
& {[m]_{1}=\left[m_{13}, m_{23}-1, m_{33}+1, m_{43}\right],} \\
& {[m]_{2}=\left[m_{13}-1, m_{23}, m_{33}+1, m_{43}\right],} \\
& {[m]_{3}=\left[m_{13}, m_{23}-1, m_{33}, m_{43}+1\right],} \\
& {[m]_{4}=\left[m_{13}-1, m_{23}, m_{33}, m_{43}+1\right],} \\
& {[m]_{5}=\left[m_{13}, m_{23}-2, m_{33}+1, m_{43}+1\right],} \\
& {[m]_{6}=\left[m_{13}-1, m_{23}-1, m_{33}+1, m_{43}+1\right]_{6},} \\
& {[m]_{7}=\left[m_{13}-2, m_{23}, m_{33}+1, m_{43}+1\right],} \\
& {[m]_{8}=\left[m_{13}-1, m_{23}-1, m_{33}+2, m_{43}\right],}
\end{aligned}
$$




$$
\begin{aligned}
& {[m]_{9}=\left[m_{13}-1, m_{23}-1, m_{33}+1, m_{43}+1\right]_{9}} \\
& {[m]_{10}=\left[m_{13}-1, m_{23}-1, m_{33}, m_{43}+2\right]} \\
& {[m]_{11}=\left[m_{13}-1, m_{23}-2, m_{33}+2, m_{43}+1\right]} \\
& {[m]_{12}=\left[m_{13}-2, m_{23}-1, m_{33}+2, m_{43}+1\right]} \\
& {[m]_{13}=\left[m_{13}-1, m_{23}-2, m_{33}+1, m_{43}+2\right]} \\
& {[m]_{14}=\left[m_{13}-2, m_{23}-1, m_{33}+1, m_{43}+2\right]} \\
& {[m]_{15}=\left[m_{13}-2, m_{23}-2, m_{33}+2, m_{43}+2\right] .}
\end{aligned}
$$

In the latest formula (3.26), with the exception of $[m]_{6}$ and $[m]_{9}$ where a degeneration is present, we skip the subscript $k$ in the r.h.s.. The proofs of (3.25) and (3.26) follow from direct computations.

Using the rule (3.11) which now reads

$$
\begin{aligned}
(m)_{k}= & \left(\frac{\left[m_{11}-m_{22}\right] !\left[m_{31}-m_{42}\right] !}{\left[m_{12}-m_{22}\right] !\left[m_{12}-m_{11}\right] !\left[m_{32}-m_{42}\right] !\left[m_{32}-m_{31}\right] !}\right)^{1 / 2} \\
& \times\left(E_{21}\right)^{m_{12}-m_{11}}\left(E_{43}\right)^{m_{32}-m_{31}}(M)_{k}
\end{aligned}
$$

we can find all the basis vectors $(m)_{k}$ :

$$
\begin{aligned}
& (m)_{0}=|0,0,0,0 ;(m)\rangle \\
& (m)_{1}=a_{1} q\left\{-\left(\frac{\left[l_{13}-l_{11}\right]\left[l_{33}-l_{31}+1\right]}{[2 l+1]\left[2 l^{\prime}+1\right]}\right)^{1 / 2}\left|1,0,0,0 ;(m)^{+11}\right\rangle\right.
\end{aligned}
$$




$$
\begin{aligned}
& -q^{l^{\prime}-s^{\prime}}\left(\frac{\left[l_{13}-l_{11}\right]\left[l_{31}-l_{43}-1\right]}{[2 l+1]\left[2 l^{\prime}+1\right]}\right)^{1 / 2}\left|0,1,0,0 ;(m)^{+11-31}\right\rangle \\
& +p^{-l+s}\left(\frac{\left[l_{11}-l_{23}\right]\left[l_{33}-l_{31}+1\right]}{[2 l+1]\left[2 l^{\prime}+1\right]}\right)^{1 / 2}|0,0,1,0 ;(m)\rangle \\
& \left.+p^{-l+s} q^{l^{\prime}-s^{\prime}}\left(\frac{\left[l_{11}-l_{23}\right]\left[l_{31}-l_{43}-1\right]}{[2 l+1]\left[2 l^{\prime}+1\right]}\right)^{1 / 2}\left|0,0,0,1 ;(m)^{-31}\right\rangle\right\}, \\
& (m)_{2}=-a_{2} q\left(\frac{q}{p}\right)^{l-s-1}\left\{\left(\frac{\left[l_{11}-l_{23}\right]\left[l_{33}-l_{31}+1\right]}{[2 l]\left[2 l^{\prime}+1\right]}\right)^{1 / 2}\left|1,0,0,0 ;(m)^{+11}\right\rangle\right. \\
& +q^{l^{\prime}-s^{\prime}}\left(\frac{\left[l_{11}-l_{23}\right]\left[l_{31}-l_{43}-1\right]}{[2 l]\left[2 l^{\prime}+1\right]}\right)^{1 / 2}\left|0,1,0,0 ;(m)^{+11-31}\right\rangle \\
& +q^{l+s+1}\left(\frac{\left[l_{13}-l_{11}\right]\left[l_{33}-l_{31}+1\right]}{[2 l]\left[2 l^{\prime}+1\right]}\right)^{1 / 2}|0,0,1,0 ;(m)\rangle \\
& \left.+q^{l+s+l^{\prime}-s^{\prime}+1}\left(\frac{\left[l_{13}-l_{11}\right]\left[l_{31}-l_{43}-1\right]}{[2 l]\left[2 l^{\prime}+1\right]}\right)^{1 / 2}\left|0,0,0,1 ;(m)^{-31}\right\rangle\right\}, \\
& (m)_{3}=a_{3}\left\{-q\left(\frac{q}{p}\right)^{l^{\prime}-s^{\prime}}\left(\frac{\left[l_{13}-l_{11}\right]\left[l_{31}-l_{43}-1\right]}{[2 l+1]\left[2 l^{\prime}\right]}\right)^{1 / 2}\left|1,0,0,0 ;(m)^{+11}\right\rangle\right. \\
& +\left(\frac{q}{p}\right)^{2 l^{\prime}}\left(\frac{\left[l_{13}-l_{11}\right]\left[l_{33}-l_{31}+1\right]}{[2 l+1]\left[2 l^{\prime}\right]}\right)^{1 / 2}\left|0,1,0,0 ;(m)^{+11-31}\right\rangle \\
& +q p^{-l+s}\left(\frac{q}{p}\right)^{l^{\prime}-s^{\prime}}\left(\frac{\left[l_{11}-l_{23}\right]\left[l_{31}-l_{43}-1\right]}{[2 l+1]\left[2 l^{\prime}\right]}\right)^{1 / 2}|0,0,1,0 ;(m)\rangle \\
& \left.-p^{-l+s}\left(\frac{q}{p}\right)^{2 l^{\prime}}\left(\frac{\left[l_{11}-l_{23}\right]\left[l_{33}-l_{31}+1\right]}{[2 l+1]\left[2 l^{\prime}\right]}\right)^{1 / 2}\left|0,0,0,1 ;(m)^{-31}\right\rangle\right\}, \\
& (m)_{4}=a_{4}\left(\frac{q}{p}\right)^{l-s+l^{\prime}-s^{\prime}-1}\left\{q\left(\frac{\left[l_{11}-l_{23}\right]\left[l_{31}-l_{43}-1\right]}{[2 l]\left[2 l^{\prime}\right]}\right)^{1 / 2}\left|1,0,0,0 ;(m)^{+11}\right\rangle\right. \\
& -p^{-l^{\prime}-s^{\prime}}\left(\frac{\left[l_{11}-l_{23}\right]\left[l_{33}-l_{31}+1\right]}{[2 l]\left[2 l^{\prime}\right]}\right)^{1 / 2}\left|0,1,0,0 ;(m)^{+11-31}\right\rangle \\
& +q^{l+s+2}\left(\frac{\left[l_{13}-l_{11}\right]\left[l_{31}-l_{43}-1\right]}{[2 l]\left[2 l^{\prime}\right]}\right)^{1 / 2}|0,0,1,0 ;(m)\rangle \\
& \left.-q^{l+s+1} p^{-l^{\prime}-s^{\prime}}\left(\frac{\left[l_{13}-l_{11}\right]\left[l_{33}-l_{31}+1\right]}{[2 l]\left[2 l^{\prime}\right]}\right)^{1 / 2}\left|0,0,0,1 ;(m)^{-31}\right\rangle\right\}, \\
& (m)_{5}=a_{5}\left(\frac{q}{p}\right)^{l^{\prime}-s^{\prime}+1}\left\{\left(\frac{\left[l_{13}-l_{11}\right]\left[l_{13}-l_{11}-1\right]}{[2 l+1][2 l+2]}\right)^{1 / 2}\left|1,1,0,0 ;(m)^{+11+11-31}\right\rangle\right.
\end{aligned}
$$




$$
\begin{aligned}
& +p^{-l+s}\left(\frac{\left[l_{13}-l_{11}\right]\left[l_{11}-l_{23}+1\right]}{[2 l+1][2 l+2]}\right)^{1 / 2}\left|0,1,1,0 ;(m)^{+11-31}\right\rangle \\
& -p^{-l+s+1}\left(\frac{\left[l_{13}-l_{11}\right]\left[l_{11}-l_{23}+1\right]}{[2 l+1][2 l+2]}\right)^{1 / 2}\left|1,0,0,1 ;(m)^{+11-31}\right\rangle \\
& \left.+p^{2(-l+s)}\left(\frac{\left[l_{11}-l_{23}\right]\left[l_{11}-l_{23}+1\right]}{[2 l+1][2 l+2]}\right)^{1 / 2}\left|0,0,1,1 ;(m)^{-31}\right\rangle\right\}, \\
& (m)_{6}=\frac{a_{6}}{a_{5}}\left(\frac{[2 l+1][2 l+2]\left[l_{13}-l_{11}\right]}{\left[l_{11}-l_{23}+1\right]}\right)^{1 / 2}(m)_{5}-a_{6}\left(\frac{q}{p}\right)^{l^{\prime}-s^{\prime}+1} \frac{[2 l+2]}{[2 l]} \times \\
& \times\left\{\left[l_{13}-l_{11}-2\right]\left(\frac{\left[l_{13}-l_{11}-1\right]}{\left[l_{11}-l_{23}+1\right]}\right)^{1 / 2}\left|1,1,0,0 ;(m)^{+11+11-31}\right\rangle\right. \\
& +p^{-l+s+1}\left[l_{13}-l_{11}-1\right]\left|0,1,1,0 ;(m)^{+11-31}\right\rangle \\
& -p^{-l+s+2}\left[l_{13}-l_{11}-1\right]\left|1,0,0,1 ;(m)^{+11-31}\right\rangle \\
& \left.+p^{-2(l-s-1)}\left(\left[l_{11}-l_{23}\right]\left[l_{13}-l_{11}\right]\right)^{1 / 2}\left|0,0,1,1 ;(m)^{-31}\right\rangle\right\}, \\
& (m)_{7}=\frac{a_{7}}{a_{5}}\left(\frac{[2 l-1][2 l][2 l+1][2 l+2]\left[l_{13}-l_{11}-1\right]\left[l_{13}-l_{11}\right]}{\left[l_{11}-l_{23}\right]\left[l_{11}-l_{23}+1\right]}\right)^{1 / 2}(m)_{5} \\
& -\frac{a_{7}}{a_{6}}[2][2 l+1]\left(\frac{[2 l-1]\left[l_{13}-l_{11}-1\right]}{[2 l]\left[l_{11}-l_{23}\right]}\right)^{1 / 2}(m)_{6} \\
& -a_{7} \frac{[2 l+1][2 l+2]}{([2 l-1][2 l])^{1 / 2}}\left(\frac{q}{p}\right)^{l^{\prime}-s^{\prime}+1}\left\{\frac{\left[l_{13}-l_{11}-2\right]\left[l_{13}-l_{11}-3\right]}{\left(\left[l_{11}-l_{23}\right]\left[l_{11}-l_{23}+1\right]\right)^{1 / 2}}\left|1,1,0,0 ;(m)^{+11+11-31}\right\rangle\right. \\
& +p^{-l+s+2}\left[l_{13}-l_{11}-2\right]\left(\frac{\left[l_{13}-l_{11}-1\right]}{\left[l_{11}-l_{23}\right]}\right)^{1 / 2}\left|0,1,1,0 ;(m)^{+11-31}\right\rangle \\
& -p^{-l+s+3}\left[l_{13}-l_{11}-2\right]\left(\frac{\left[l_{13}-l_{11}-1\right]}{\left[l_{11}-l_{23}\right]}\right)^{1 / 2}\left|1,0,0,1 ;(m)^{+11-31}\right\rangle \\
& \left.+p^{-2(l-s-2)}\left(\left[l_{13}-l_{11}-1\right]\left[l_{13}-l_{11}\right]\right)^{1 / 2}\left|0,0,1,1 ;(m)^{-31}\right\rangle\right\}, \\
& (m)_{8}=a_{8} q^{2}\left(\frac{q}{p}\right)^{l-s-1}\left\{\left(\frac{\left[l_{33}-l_{31}+1\right]\left[l_{33}-l_{31}+2\right]}{\left[2 l^{\prime}+1\right]\left[2 l^{\prime}+2\right]}\right)^{1 / 2}\left|1,0,1,0 ;(m)^{+11}\right\rangle\right. \\
& +q^{2 l^{\prime}}\left(\frac{\left[l_{33}-l_{31}+2\right]\left[l_{31}-l_{43}-1\right]}{\left[2 l^{\prime}+1\right]\left[2 l^{\prime}+2\right]}\right)^{1 / 2}\left|1,0,0,1 ;(m)^{+11-31}\right\rangle \\
& +q^{2 l^{\prime}+1}\left(\frac{\left[l_{33}-l_{31}+2\right]\left[l_{31}-l_{43}-1\right]}{\left[2 l^{\prime}+1\right]\left[2 l^{\prime}+2\right]}\right)^{1 / 2}\left|0,1,1,0 ;(m)^{+11-31}\right\rangle
\end{aligned}
$$




$$
\begin{aligned}
& \left.+q^{2\left(2 l^{\prime}+1\right)}\left(\frac{\left[l_{31}-l_{43}-2\right]\left[l_{31}-l_{43}-1\right]}{\left[2 l^{\prime}+1\right]\left[2 l^{\prime}+2\right]}\right)^{1 / 2}\left|0,1,0,1 ;(m)^{+11-31-31}\right\rangle\right\}, \\
& (m)_{9}=\frac{a_{9}}{a_{8}}\left(\frac{\left[2 l^{\prime}+1\right]\left[2 l^{\prime}+2\right]\left[l_{33}-l_{31}+2\right]}{\left[l_{31}-l_{43}-1\right]}\right)^{1 / 2}(m)_{8} \\
& -a_{9} q^{2}\left(\frac{q}{p}\right)^{l-s-1} \frac{\left[2 l^{\prime}+2\right]}{\left[2 l^{\prime}\right]}\left\{\left[l_{33}-l_{31}\right]\left(\frac{\left[l_{33}-l_{31}+1\right]}{\left[l_{31}-l_{43}-1\right]}\right)^{1 / 2}\left|1,0,1,0 ;(m)^{+11}\right\rangle\right. \\
& +q^{l^{\prime}-s^{\prime}-1}\left[l_{33}-l_{31}+1\right]\left|1,0,0,1 ;(m)^{+11-31}\right\rangle \\
& +q^{l^{\prime}-s^{\prime}}\left[l_{33}-l_{31}+1\right]\left|0,1,1,0 ;(m)^{+11-31}\right\rangle \\
& \left.+q^{2\left(l^{\prime}-s^{\prime}\right)}\left(\left[l_{33}-l_{31}+2\right]\left[l_{31}-l_{43}-2\right]\right)^{1 / 2}\left|0,1,0,1 ;(m)^{+11-31-31}\right\rangle\right\} \\
& (m)_{10}=\frac{a_{10}}{a_{8}}\left(\frac{\left[2 l^{\prime}-1\right]\left[2 l^{\prime}\right]\left[2 l^{\prime}+1\right]\left[2 l^{\prime}+2\right]\left[l_{33}-l_{31}+1\right]\left[l_{33}-l_{31}+2\right]}{\left[l_{31}-l_{43}-2\right]\left[l_{31}-l_{43}-1\right]}\right)^{1 / 2}(m)_{8} \\
& -\frac{a_{10}}{a_{9}}[2]\left[2 l^{\prime}+1\right]\left(\frac{\left[2 l^{\prime}-1\right]\left[l_{33}-l_{31}+1\right]}{\left[2 l^{\prime}\right]\left[l_{31}-l_{43}-2\right]}\right)^{1 / 2}(m)_{9} \\
& -a_{10}\left(\frac{q}{p}\right)^{l-s-1} \frac{\left[2 l^{\prime}+1\right]\left[2 l^{\prime}+2\right]}{\left[2 l^{\prime}-1\right]\left[2 l^{\prime}\right]}\left\{q^{2}\left[l_{33}-l_{31}-1\right]\left[l_{33}-l_{31}\right] \times\right. \\
& \times\left(\frac{\left[2 l^{\prime}-1\right]\left[2 l^{\prime}\right]}{\left[l_{31}-l_{43}-2\right]\left[l_{31}-l_{43}-1\right]}\right)^{1 / 2}\left|1,0,1,0 ;(m)^{+11}\right\rangle \\
& +q^{l^{\prime}-s^{\prime}}\left[l_{33}-l_{31}\right]\left(\frac{\left[2 l^{\prime}-1\right]\left[2 l^{\prime}\right]\left[l_{33}-l_{31}+1\right]}{\left[l_{31}-l_{43}-2\right]}\right)^{1 / 2}\left|1,0,0,1 ;(m)^{+11-31}\right\rangle \\
& +q^{l^{\prime}-s^{\prime}+1}\left[l_{33}-l_{31}\right]\left(\frac{\left[2 l^{\prime}-1\right]\left[2 l^{\prime}\right]\left[l_{33}-l_{31}+1\right]}{\left[l_{31}-l_{43}-2\right]}\right)^{1 / 2}\left|0,1,1,0 ;(m)^{+11-31}\right\rangle \\
& \left.+q^{2\left(l^{\prime}-s^{\prime}\right)}\left(\left[2 l^{\prime}-1\right]\left[2 l^{\prime}\right]\left[l_{33}-l_{31}+1\right]\left[l_{33}-l_{31}+2\right]\right)^{1 / 2}\left|0,1,0,1 ;(m)^{+11-31-31}\right\rangle\right\}, \\
& (m)_{11}=a_{11}\left(\frac{q}{p}\right)^{l-s+l^{\prime}-s^{\prime}+1}\left\{p\left(\frac{\left[l_{13}-l_{11}-1\right]\left[l_{33}-l_{31}+2\right]}{[2 l+1]\left[2 l^{\prime}+1\right]}\right)^{1 / 2}\left|1,1,1,0 ;(m)^{+11+11-31}\right\rangle\right. \\
& +p^{-l+s+2}\left(\frac{\left[l_{11}-l_{23}+1\right]\left[l_{33}-l_{31}+2\right]}{[2 l+1]\left[2 l^{\prime}+1\right]}\right)^{1 / 2}\left|1,0,1,1 ;(m)^{+11-31}\right\rangle \\
& +q^{l^{\prime}-s^{\prime}+1} p\left(\frac{\left[l_{13}-l_{11}-1\right]\left[l_{31}-l_{43}-2\right]}{[2 l+1]\left[2 l^{\prime}+1\right]}\right)^{1 / 2}\left|1,1,0,1 ;(m)^{+11+11-31-31}\right\rangle \\
& \left.+q^{l^{\prime}-s^{\prime}+2} p^{-l+s+1}\left(\frac{\left[l_{11}-l_{23}+1\right]\left[l_{31}-l_{43}-2\right]}{[2 l+1]\left[2 l^{\prime}+1\right]}\right)^{1 / 2}\left|0,1,1,1 ;(m)^{+11-31-31}\right\rangle\right\} \text {, }
\end{aligned}
$$




$$
\begin{aligned}
(m)_{12}= & \frac{a_{12}}{a_{11}}\left(\frac{[2 l][2 l+1]\left[l_{13}-l_{11}-1\right]}{\left[l_{11}-l_{23}+1\right]}\right)^{1 / 2}(m)_{11} \\
& -a_{12} p\left(\frac{q}{p}\right)^{l-s+l^{\prime}-s^{\prime}} \frac{[2 l+1]}{[2 l]}\left\{\left[l_{13}-l_{11}-2\right]\left(\frac{[2 l]\left[l_{33}-l_{31}+2\right]}{\left[l_{11}-l_{23}+1\right]\left[2 l^{\prime}+1\right]}\right)^{1 / 2} \times\right. \\
& \times\left|1,1,1,0 ;(m)^{+11+11-31}\right\rangle \\
& +p^{-l+s+2}\left(\frac{[2 l]\left[l_{13}-l_{11}-1\right]\left[l_{33}-l_{31}+2\right]}{\left[2 l^{\prime}+1\right]}\right)^{1 / 2}\left|1,0,1,1 ;(m)^{+11-31}\right\rangle \\
& +q^{l^{\prime}-s^{\prime}+1}\left[l_{13}-l_{11}-2\right]\left(\frac{[2 l]\left[l_{31}-l_{43}-2\right]}{\left[\left[l_{11}-l_{23}+1\right]\right]\left[2 l^{\prime}+1\right]}\right)^{1 / 2}\left|1,1,0,1 ;(m)^{+11+11-31-31}\right\rangle \\
& \left.+q^{l^{\prime}-s^{\prime}+2} p^{-l+s+1}\left(\frac{[2 l]\left[l_{13}-l_{11}-1\right]\left[l_{31}-l_{43}-2\right]}{\left[2 l^{\prime}+1\right]}\right)^{1 / 2}\left|0,1,1,1 ;(m)^{+11-31-31}\right\rangle\right\},
\end{aligned}
$$

$$
\begin{aligned}
(m)_{13}= & \frac{a_{13}}{a_{11}}\left(\frac{\left[2 l^{\prime}\right]\left[2 l^{\prime}+1\right]\left[l_{33}-l_{31}+2\right]}{\left[l_{31}-l_{43}-2\right]}\right)^{1 / 2}(m)_{11} \\
& -a_{13} p\left(\frac{q}{p}\right)^{l-s+l^{\prime}-s^{\prime}} \frac{\left[2 l^{\prime}+1\right]}{\left[2 l^{\prime}\right]}\left\{\left[l_{33}-l_{31}+1\right]\left(\frac{\left[2 l^{\prime}\right]\left[l_{13}-l_{11}-1\right]}{[2 l+1]\left[l_{31}-l_{43}-2\right]}\right)^{1 / 2} \times\right. \\
& \times\left|1,1,1,0 ;(m)^{+11+11-31}\right\rangle \\
& +p^{-l+s+1}\left[l_{33}-l_{31}+1\right]\left(\frac{\left[l_{11}-l_{23}+1\right]\left[2 l^{\prime}\right]}{[2 l+1]\left[l_{31}-l_{43}-2\right]}\right)^{1 / 2}\left|1,0,1,1 ;(m)^{+11-31}\right\rangle \\
& +q^{l^{\prime}-s^{\prime}}\left(\frac{\left[l_{13}-l_{11}-1\right]\left[l_{33}-l_{31}+2\right]\left[2 l^{\prime}\right]}{[2 l+1]}\right)^{1 / 2}\left|1,1,0,1 ;(m)^{+11+11-31-31}\right\rangle \\
& \left.+q^{l^{\prime}-s^{\prime}+1} p^{-l+s}\left(\frac{\left[l_{11}-l_{23}+1\right]\left[l_{33}-l_{31}+2\right]\left[2 l^{\prime}\right]}{[2 l+1]}\right)^{1 / 2}\left|0,1,1,1 ;(m)^{+11-31-31}\right\rangle\right\}
\end{aligned}
$$

$$
\begin{aligned}
(m)_{14}= & \frac{a_{14}}{a_{11}}\left(\frac{[2 l][2 l+1]\left[l_{13}-l_{11}-1\right]\left[2 l^{\prime}\right]\left[2 l^{\prime}+1\right]\left[l_{33}-l_{31}+2\right]}{\left[l_{11}-l_{23}+1\right]\left[l_{31}-l_{43}-2\right]}\right)^{1 / 2}(m)_{11} \\
& -\frac{a_{14}}{a_{12}}\left(\frac{\left[2 l^{\prime}\right]\left[2 l^{\prime}+1\right]\left[l_{33}-l_{31}+2\right]}{\left[l_{31}-l_{43}-2\right]}\right)^{1 / 2}(m)_{12} \\
& -\frac{a_{14}}{a_{13}}\left(\frac{[2 l][2 l+1]\left[l_{13}-l_{11}-1\right]}{\left[l_{11}-l_{23}+1\right]}\right)^{1 / 2}(m)_{13} \\
& -a_{14} p\left(\frac{q}{p}\right)^{l-s+l^{\prime}-s^{\prime}-1} \frac{[2 l+1]\left[2 l^{\prime}+1\right]}{[2 l]\left[2 l^{\prime}\right]}\left\{\left[l_{13}-l_{11}-2\right]\left[l_{33}-l_{31}+1\right] \times\right. \\
& \times\left(\frac{[2 l]\left[2 l^{\prime}\right]}{\left[l_{11}-l_{23}+1\right]\left[l_{31}-l_{43}-2\right]}\right)^{1 / 2}\left|1,1,1,0 ;(m)^{+11+11-31}\right\rangle
\end{aligned}
$$




$$
\begin{aligned}
& +p^{-l+s+2}\left[l_{33}-l_{31}+1\right]\left(\frac{\left[l_{13}-l_{11}-1\right][2 l]\left[2 l^{\prime}\right]}{\left[l_{31}-l_{43}-2\right]}\right)^{1 / 2}\left|1,0,1,1 ;(m)^{+11-31}\right\rangle \\
& +q^{l^{\prime}-s^{\prime}}\left[l_{13}-l_{11}-2\right]\left(\frac{[2 l]\left[2 l^{\prime}\right]\left[l_{33}-l_{31}+2\right]}{\left[l_{11}-l_{23}+1\right]}\right)^{1 / 2}\left|1,1,0,1 ;(m)^{+11+11-31-31}\right\rangle \\
& \left.+q^{l^{\prime}-s^{\prime}+1} p^{-l+s+1}\left([2 l]\left[l_{13}-l_{11}-1\right]\left[l_{33}-l_{31}+2\right]\left[2 l^{\prime}\right]\right)^{1 / 2}\left|0,1,1,1 ;(m)^{+11-31-31}\right\rangle\right\} \\
(m)_{15}= & a_{15}(m)
\end{aligned}
$$

where $l=\frac{1}{2}\left(m_{13}-m_{23}\right), s=m_{11}-\frac{1}{2}\left(m_{13}+m_{23}\right), l^{\prime}=\frac{1}{2}\left(m_{33}-m_{43}\right)$ and $s^{\prime}=$ $m_{31}-\frac{1}{2}\left(m_{33}+m_{43}\right)$, while $(m)_{k}^{ \pm i j}$ is a GZ basis vector obtained from $(m)_{k}$ with replacing the element $m_{i j}$ by $m_{i j} \pm 1$. We can write down the coefficients in $(3.27)$ all in terms of $l, s, l^{\prime}$ and $s^{\prime}$ only but here we leave them partially expressed in terms of $l_{i j}$ and $l_{i j}^{\prime}$. From (3.27) we can immediately find all the (local) lowest weight vectors $(M)_{k}^{V}$ which, by definition, are annihilated by $E_{21}$ and $E_{43}$. Let us remind again that every firdirmod $V_{k}^{p, q}$ on a level $\eta$, spanned on linear combinations of $\left|\theta_{1}, \theta_{2}, \theta_{3}, \theta_{4} ;(m)\right\rangle$ in (3.14) with a fixed $\sum_{i=1}^{4} \theta_{i} \equiv \eta$ is a linear space of homogeneous polynomials of a definite power $\eta$ in the negative odd generators $E_{i j}$ $(1 \leq j<3 \leq i \leq 4)$ acting on $(m) \in V_{0}^{p, q}([m])$. Taking into account all results obtained above we have proved the following assertion

Proposition 3 : Every $U_{p, q}\left[g l(2 / 2)_{0}\right]$-fidirmod $V_{k}^{p, q}$ in decomposition (3.16) is characterized by a highest weight $[m]_{k}$ given in (3.25) and is spanned by a GZ basis $(m)_{k}$ given in (3.27).

The latest formula (3.27), in fact, represents a way in which the reduced basis 
is expressed in terms of the induced basis and vas versa it is not a problem for us to find the inverse relation between these bases (see the Appendix). For further convenience the vectors $(m)_{\tilde{k}} \equiv(m)_{k}($ for $k=6,7,9,10,12,13$ and 14) are partially given via other $(m)_{k}$ which are completely expressed in terms of $\left|\theta_{1}, \theta_{2}, \theta_{3}, \theta_{4} ;(m)\right\rangle$. It is not difficult to write down the explicit decompositions of these $(m)_{\tilde{k}}$ in the induced basis. But here we prefer the expressions in (3.27) which are more compact and more convenient for finding the inverse relation between two bases and matrix elements of odd generators.

Now we are ready to canculate the matrix elements of the generators $E_{i j}$. It is sufficient to canculate the matrix elements of the Cartan-Chevalley generators only, since $U_{p, q}[g l(2 / 2)]$ can be generated by these generators and any its representation in some basis is completely defined by their actions on the same basis. For the even generators which do not shift the $\eta$ 's we readily have

$$
\begin{aligned}
E_{11}(m)_{k} & =\left(l_{11}+1\right)(m)_{k}, \\
E_{22}(m)_{k} & =\left(l_{12}+l_{22}-l_{11}+2\right)(m)_{k}, \\
E_{12}(m)_{k} & =\left(\left[l_{12}-l_{11}\right]\left[l_{11}-l_{22}\right]\right)^{1 / 2}(m)_{k}^{+11}, \\
E_{21}(m)_{k} & =\left(\left[l_{12}-l_{11}+1\right]\left[l_{11}-l_{22}-1\right]\right)^{1 / 2}(m)_{k}^{-11} \\
L_{1}(m)_{k} & =\frac{1}{2}\left(l_{12}-l_{22}-1\right)(m)_{k}, \\
E_{33}(m)_{k} & =\left(l_{31}+1\right)(m)_{k}, \\
E_{44}(m)_{k} & =\left(l_{32}+l_{42}-l_{31}+2\right)(m)_{k}, \\
E_{34}(m)_{k} & =\left(\left[l_{32}-l_{31}\right]\left[l_{31}-l_{42}\right]\right)^{1 / 2}(m)_{k}^{+31},
\end{aligned}
$$




$$
\begin{aligned}
E_{43}(m)_{k} & =\left(\left[l_{32}-l_{31}+1\right]\left[l_{31}-l_{42}-1\right]\right)^{1 / 2}(m)_{k}^{-31} \\
L_{3}(m)_{k} & =\frac{1}{2}\left(l_{32}-l_{42}-1\right)(m)_{k} .
\end{aligned}
$$

As the matrix elements of $E_{23}$ and $E_{32}$ are very long expressions we only explain here how to find them. By construction a reduced basis vector $(m)_{k}$ in $(3.27)$ belonging to a fidirmod $V_{k}^{p, q}$ on a level $\eta$ is a homogeneous polynomial of a power $\eta$ in odd generators $E_{i j}, 1 \leq j<3 \leq i \leq 4$ acting on $(m) \in V_{0}^{p, q}$. Under the action of $E_{23}$ (or $E_{32}$, respectively) this vector $(m)_{k}$ is shifted to other fidirmods $V_{k^{\prime}}^{p, q}$ on the previous level $\eta-1$ (or on the next level $\eta+1$, respectively), i.e., we get on the r.h.s of $E_{23}(m)_{k}$ (or $E_{32}(m)_{k}$, respectively) a homogeneous polynomial of a degree $\eta-1$ (or $\eta+1$, respectively). Using the inverse relations (A.1) we can express the latter polynomials obtained in terms of the reduced basis, that is we get matrix elements of $E_{23}$ and $E_{32}$ in this basis. It is a standard way to find matrix elements but in practice we can use a trick making calculations simpler. Since $E_{23}$ commutes with $E_{21}$ and $E_{43}$ we first calculate the action of $E_{23}$ on the highest vectors only and then apply (3.11) to find all matrix elements of this generator on arbitrary $(m)_{k}$. It is less complicated to compute matrix elements of $E_{32}$ in the standard way but we can apply a similar trick, namely, we first calculate the action of $E_{23}$ (which commutes with $E_{12}$ and $\left.E_{34}\right)$ on the lowest weight vectors and then apply the rule inverse to (3.11).

It can be shown that the representations constructed contain all finite-dimensional irreducible representations of $U_{p, q}[g l(2 / 2)]$ classified as typical or nontypical repre- 
sentations which are subjects of next investigations.

\section{Conclusion}

We have considered the two-parametric quantum deformations $U_{p, q}[g l(2 / 2)]$ and described in detail a method for constructing its finite-dimensional representations. The representations constructed can be decomposed into finite-dimensional irreducible representations of the even subalgebra $U_{p, q}\left[g l(2 / 2)_{0}\right]$ and therefore can be given in bases of the latter. Using Poincaré-Birkhoff-Witt theorem and the induced representation method we constructed the induced basis of the induced module $W^{p, q}$. This basis, however, does not allow a clear description of a decomposition of $W^{p, q}$ into $U_{p, q}\left[g l(2 / 2)_{0}\right]$-fidirmods. It was the reason the reduced basis was introduced. The latter basis representing a union of GZ bases of the even subalgebra $U_{p, q}\left[g l(2 / 2)_{0}\right]$ according to the branching rule $U_{p, q}[g l(2 / 2)] \supset U_{p, q}\left[g l(2 / 2)_{0}\right] \supset$ $g l(1) \otimes g l(1)$ is refered to as Quasi-GZ basis. This step is intermediate but of independent interest. Having these two bases, the induced and the reduced ones, and the relations between them we can find all matrix elements of finite-dimensional representations of $U_{p, q}[g l(2 / 2)]$. It turn out that the representations constructed contain all finite-dimensional irreducible representations of $U_{p, q}[g l(2 / 2)]$ and can be classified into typical and nontypical representations which are subjects of later papers.

Looking at the basis transformations and the matrix elements we observe, even at generic deformation parameters, some "anomalies" which are canceled out at 
$p=q$. It means that the finite-dimensional representations of the two-parametric quantum superalgebra $U_{q}[g l(2 / 2)]$ are not simply trivial deformations from those of the classical Lie superalgebra $g l(2 / 2)$ in the sense that they can not be found from classical analogues by putting quantum deformation brackets in appropriate places unlike many cases of one-parametric deformations. For example, the expressions

$$
\frac{q}{p}[2 l]\left[l_{13}-l_{11}\right]-[2 l+1]\left[l_{13}-l_{11}-1\right]
$$

and

$$
\frac{q}{p}\left[2 l^{\prime}\right]\left[l_{33}-l_{31}\right]-\left[2 l^{\prime}+1\right]\left[l_{33}-l_{31}-1\right]
$$

appearing in the basis transformations and matrix elements can be written in the forms:

$$
\left(\frac{q}{p}-1\right)[2 l]\left[l_{13}-l_{11}\right]+\left(\frac{q}{p}\right)^{l_{13}-l_{11}-1}\left[l_{11}-l_{23}\right]
$$

and

$$
\left(\frac{q}{p}-1\right)\left[2 l^{\prime}\right]\left[l_{33}-l_{31}\right]+\left(\frac{q}{p}\right)^{l_{33}-l_{31}-1}\left[l_{31}-l_{43}\right]
$$

respectively. At $p=q$ the latest expressions become $\left[l_{11}-l_{23}\right]$ and $\left[l_{31}-l_{43}\right]$, respectively, exactly as in the one-parametric case [22, 23].

We hope that it is not very difficult to extend the present method to the case of one or both deformation parameters being roots of unity. For conclusion, let us emphasize that our method has an advantage that it avoids the use of the ClebschGordan coefficients which are not always known, especially for higher rank (classical and quantum) groups and multi-parametric deformations. 


\section{Acknowledgements}

I would like to thank the Nishina memorial foundation for financial support and the Department of Physics, Chuo University, Tokyo, Japan for warm hospitality. Fruitful discussions with K. Furuta, T. Inami and other members of the Theory Group of the Department of Physics, Chuo University, are also hereby acknowledged.

This work was supported in part by the National Research Programme for Natural Sciences of Vietnam under grant number KT - 04.1.1. 


\section{References}

[1] M. Jimbo ed., Yang-Baxter equation in intergrable systems (World Scientific, Singapore 1989).

[2] L. D. Faddeev, N. Yu. Reshetikhin and L. A. Takhtajan, Algebra and Analys, 1, 178 (1987).

[3] V. D. Drinfel'd, "Quantum groups", in Proceedings of the International Congress of Mathematicians, 1986, Berkeley (The American Mathematical Society, Providence, RI, 1987), Vol. 1, pp. 798-820 .

[4] Yu. I. Manin, Quantum groups and non-commutative geometry (Centre des Recherchers Mathématiques, Montréal, 1988).

[5] Yu. I. Manin, Topics in non-commutative geometry (Princeton University Press, Princeton, New Jersey, 1991).

[6] M. Jimbo, Lett. Math. Phys. 10, 63 (1985), ibit 11, 247 (1986).

[7] S. I. Woronowicz, Comm. Math. Phys., 111, 613 (1987).

[8] C. Gómez, M. Ruiz-Altaba and G. Sierra, Quantum groups in two-dimensional physics (Cambridge University Press, Cambridge, 1996).

[9] S. Majid, Foundation of quantum group theory (Cambridge University Press, Cambridge, 1995).

[10] V. Chari and A. Pressley, A guide to quantum groups (Cambridge University Press, Cambridge, 1994). 
[11] Ch. Kassel, Quantum groups (Springer - Verlag, New York, 1995).

[12] C. N. Yang and M. L. Ge eds., Braid groups, knot theory and statistical mechanics, (World Scientific, Singapore, 1989).

[13] H. D. Doebner and J. D. Hennig eds., Quantum groups, Lecture Notes in Physics (Springer - Verlag, Berlin 1990), Vol. 370.

[14] P. P. Kulish ed., Quantum groups, Lecture Notes in Mathematics (Springer Verlag, Berlin 1992), Vol. 1510.

[15] , Nguyen Anh Ky, Superalgebras, their quantum deformations and the induced representations method, math.QA/9810170 and references therein.

[16] E. Celeghini, Tch. Palev and M. Tarlini, Mod. Phys. Lett. B 5, 187 (1991).

[17] Tch. D. Palev and V. N. Tolstoy, Comm. Math. Phys. 141, 549 (1991).

[18] Yu. I. Manin, Comm. Math. Phys. 123, 163 (1989).

[19] M. Chaichian and P. Kulish, Phys. Lett. B 234, 72 (1990).

[20] R. Floreanini, V. Spiridonov and L. Vinet, Comm. Math. Phys. 137, 149 (1991);

E. D'Hoker, R. Floreanini and L. Vinet, J. Math. Phys., 32, 1427 (1991).

[21] R. B. Zhang, J. Math. Phys., 34, 1236 (1993).

[22] Nguyen Anh Ky, J. Math. Phys. 35, 2583 (1994), hep-th/9305183.

[23] Nguyen Anh Ky and N. Stoilova, J. Math. Phys. 36, 5979 (1995) and hepth/9411098. 
[24] V. K. Dobrev and E. H. Tahri, J. Phys. A: Math. Gen., 32, 4209 (1999).

[25] Nguyen Anh Ky, J. Phys. A: Math. Gen., 29, 1541 (1996) or math.QA/9909067.

[26] R. Zhang, J. Phys. A: Math. Gen., 23, 817 (1994).

[27] R. Floreanini, D. Leites and L. Vinet, Lett. Math. Phys. 23, 127 (1991).

[28] M. Scheunert, Lett. Math. Phys. 24, 173 (1992).

[29] S. M. Khoroshkin and V. N. Tolstoy, Comm. Math. Phys. 141, 599 (1991).

[30] N. Berkovits, C. Vafa and E. Witten, Conformal field theory of AdS background with Ramond-Ramond flux, hep-th/9902098; M. Bershadsky, S. Zhukov and A. Vaintrob, $P S L(n / n)$ sigma model as a conformal field theory, hep-th/9902180.

[31] A. H. Kamupingene, Nguyen Anh Ky and Tch. D. Palev, J. Math. Phys. 30, 553 (1989); Tch. Palev and N. Stoilova, J. Math. Phys., 31, 953 (1990).

[32] Nguyen Anh Ky, "On the algebraic relation between one-parametric and multiparametric quantum superalgebras", in Proceedings of the 22-th national workshop on theoretical physics, Doson, 3-5 August 1997 (Institute of Physics, Hanoi, 1998), pp. 24-28. 


\section{Appendix}

The induced basis (4.20) is expressed in terms of the reduced basis through the following inverse relation

$$
\begin{aligned}
& |1,0,0,0 ;(m)\rangle=-\frac{1}{a_{1}} q^{l+s-1} p^{-l^{\prime}-s^{\prime}}\left(\frac{\left[l_{13}-l_{11}+1\right]\left[l_{33}-l_{31}+1\right]}{[2 l+1]\left[2 l^{\prime}+1\right]}\right)^{1 / 2}(m)_{1}^{-11} \\
& -\frac{1}{a_{2}} \frac{q^{-l+s-1} p^{-l^{\prime}-s^{\prime}-1}}{[2 l+1]}\left(\frac{[2 l]\left[l_{11}-l_{23}-1\right]\left[l_{33}-l_{31}+1\right]}{\left[2 l^{\prime}+1\right]}\right)^{1 / 2}(m)_{2}^{-11} \\
& -\frac{1}{a_{3}} \frac{q^{l+s} p^{l^{\prime}-s^{\prime}}}{\left[2 l^{\prime}+1\right]}\left(\frac{\left[l_{13}-l_{11}+1\right]\left[l_{31}-l_{43}-1\right]\left[2 l^{\prime}\right]}{[2 l+1]}\right)^{1 / 2}(m)_{3}^{-11} \\
& +\frac{1}{a_{4}} \frac{q^{-l+s} p^{l^{\prime}-s^{\prime}-1}}{[2 l+1]\left[2 l^{\prime}+1\right]}\left([2 l]\left[l_{11}-l_{23}-1\right]\left[2 l^{\prime}\right]\left[l_{31}-l_{43}-1\right]\right)^{1 / 2}(m)_{4}^{-11}, \\
& |0,1,0,0 ;(m)\rangle=-\frac{1}{a_{1}} q^{l+s}\left(\frac{\left[l_{13}-l_{11}+1\right]\left[l_{31}-l_{43}\right]}{[2 l+1]\left[2 l^{\prime}+1\right]}\right)^{1 / 2}(m)_{1}^{-11+31} \\
& -\frac{1}{a_{2}} \frac{q^{-l+s}}{p[2 l+1]}\left(\frac{[2 l]\left[l_{11}-l_{23}-1\right]\left[l_{31}-l_{43}\right]}{\left[2 l^{\prime}+1\right]}\right)^{1 / 2}(m)_{2}^{-11+31} \\
& +\frac{1}{a_{3}}\left(\frac{p}{q}\right)^{l^{\prime}-s^{\prime}-1} \frac{q^{l+s}}{\left[2 l^{\prime}+1\right]}\left(\frac{\left[l_{13}-l_{11}+1\right]\left[2 l^{\prime}\right]\left[l_{33}-l_{31}\right]}{[2 l+1]}\right)^{1 / 2}(m)_{3}^{-11+31} \\
& -\frac{1}{a_{4}}\left(\frac{p}{q}\right)^{l^{\prime}-s^{\prime}-2} \frac{q^{-l+s-1}}{[2 l+1]\left[2 l^{\prime}+1\right]}\left(\frac{[2 l]\left[l_{11}-l_{23}-1\right]\left[2 l^{\prime}\right]}{\left[l_{33}-l_{31}\right.}\right)^{1 / 2}(m)_{4}^{-11+31} \text {, } \\
& |0,0,1,0 ;(m)\rangle=-\frac{1}{a_{1}} q^{-1} p^{-l^{\prime}-s^{\prime}}\left(\frac{\left[l_{11}-l_{23}\right]\left[l_{33}-l_{31}+1\right]}{[2 l+1]\left[2 l^{\prime}+1\right]}\right)^{1 / 2}(m)_{1} \\
& +\frac{1}{a_{2}}\left(\frac{p}{q}\right)^{l-s} \frac{p^{-l^{\prime}-s^{\prime}-1}}{[2 l+1]}\left(\frac{[2 l]\left[l_{13}-l_{11}\right]\left[l_{33}-l_{31}+1\right]}{\left[2 l^{\prime}+1\right]}\right)^{1 / 2}(m)_{2} \\
& +\frac{1}{a_{3}} \frac{p^{l^{\prime}-s^{\prime}}}{\left[2 l^{\prime}+1\right]}\left(\frac{\left[l_{11}-l_{23}\right]\left[2 l^{\prime}\right]\left[l_{31}-l_{43}-1\right]}{[2 l+1]}\right)^{1 / 2}(m)_{3} \\
& +\frac{1}{a_{4}}\left(\frac{p}{q}\right)^{l-s-1} \frac{p^{l^{\prime}-s^{\prime}}}{[2 l+1]\left[2 l^{\prime}+1\right]} \times \\
& \times\left([2 l]\left[l_{13}-l_{11}\right]\left[2 l^{\prime}\right]\left[l_{31}-l_{43}-1\right]\right)^{1 / 2}(m)_{4}, \\
& |0,0,0,1 ;(m)\rangle=\frac{1}{a_{1}}\left(\frac{\left[l_{11}-l_{23}\right]\left[l_{31}-l_{43}+1\right]}{[2 l+1]\left[2 l^{\prime}+1\right]}\right)^{1 / 2}(m)_{1}^{+31}
\end{aligned}
$$




$$
\begin{aligned}
& -\frac{1}{a_{2}}\left(\frac{p}{q}\right)^{l-s-1} \frac{1}{[2 l+1]}\left(\frac{[2 l]\left[l_{13}-l_{11}\right]\left[l_{31}-l_{43}\right]}{\left[2 l^{\prime}+1\right]}\right)^{1 / 2}(m)_{2}^{+31} \\
& -\frac{1}{a_{3}}\left(\frac{p}{q}\right)^{l^{\prime}-s^{\prime}-1} \frac{1}{\left[2 l^{\prime}+1\right]}\left(\frac{\left[l_{11}-l_{23}\right]\left[2 l^{\prime}\right]\left[l_{33}-l_{31}\right]}{[2 l+1]}\right)^{1 / 2}(m)_{3}^{+31} \\
& -\frac{1}{a_{4}}\left(\frac{p}{q}\right)^{l-s+l^{\prime}-s^{\prime}-2} \frac{1}{[2 l+1]\left[2 l^{\prime}+1\right]} \times \\
& \times\left(\frac{[2 l]\left[2 l^{\prime}\right]}{\left[l_{13}-l_{11}\right]\left[l_{31}-l_{43}\right]}\right)^{1 / 2}(m)_{4}^{+31} \\
& |1,1,0,0 ;(m)\rangle=\frac{1}{a_{5}} q^{2(l+s)}\left(\frac{p}{q}\right)^{l^{\prime}-s^{\prime}}\left(\frac{\left[l_{13}-l_{11}+1\right]\left[l_{13}-l_{11}+2\right]}{[2 l+1][2 l+2]}\right)^{1 / 2}(m)_{5}^{-11-11+31} \\
& +\frac{1}{a_{6}}\left(\frac{p}{q}\right)^{l^{\prime}-s^{\prime}-3} \frac{q^{-2(l-s)-3}}{[2 l+2]}\left(p^{2} q^{2 l-1}+(p-q)[2 l-1]\right) \times \\
& \times\left(\left[l_{11}-l_{23}-1\right]\left[l_{13}-l_{11}-1\right]\right)^{1 / 2}(m)_{6}^{-11-11+31} \\
& -\frac{1}{a_{7}}\left(\frac{p}{q}\right)^{l^{\prime}-s^{\prime}-3} \frac{q^{-2(l-s+1)}}{[2][2 l+1][2 l+2]} \times \\
& \times\left([2 l][2 l-1]\left[l_{11}-l_{23}-2\right]\left[l_{11}-l_{23}-1\right]\right)^{1 / 2}(m)_{7}^{-11-11+31}, \\
& E^{(-2)} \equiv|0,1,1,0 ;(m)\rangle-p|1,0,0,1 ;(m)\rangle \\
& =\frac{1}{a_{5}} q^{l+s+1}\left(\frac{p}{q}\right)^{l^{\prime}-s^{\prime}+1}[2]\left(\frac{\left[l_{13}-l_{11}+1\right]\left[l_{11}-l_{23}\right]}{[2 l+1][2 l+2]}\right)^{1 / 2}(m)_{5}^{-11+31} \\
& +\frac{1}{a_{6}}\left(\frac{p}{q}\right)^{l-s+l^{\prime}-s^{\prime}-2} \frac{q^{-l+s}}{[2 l+2]}\left\{[2][2 l-1]\left[l_{13}-l_{11}\right]\right. \\
& \left.-[2 l]\left(p^{2}\left[l_{13}-l_{11}\right]+q^{-1}\left[l_{13}-l_{11}-1\right]\right)\right\}(m)_{6}^{-11+31} \\
& +\frac{1}{a_{7}}\left(\frac{p}{q}\right)^{l-s+l^{\prime}-s^{\prime}-2} \frac{q^{-l+s}}{[2 l+1][2 l+2]} \times \\
& \times\left([2 l][2 l-1]\left[l_{13}-l_{11}\right]\left[l_{11}-l_{23}-1\right]\right)^{1 / 2}(m)_{7}^{-11+31}, \\
& |0,0,1,1 ;(m)\rangle=\frac{1}{a_{5}}\left(\frac{p}{q}\right)^{l^{\prime}-s^{\prime}}\left(\frac{\left[l_{11}-l_{23}\right]\left[l_{11}-l_{23}+1\right]}{[2 l+1][2 l+2]}\right)^{1 / 2}(m)_{5}^{+31} \\
& +\frac{1}{a_{6}}\left(\frac{p}{q}\right)^{2(l-s)+l^{\prime}-s^{\prime}-4}\left\{\frac{p}{q}[2 l]\left[l_{13}-l_{11}-2\right]-[2 l-1]\left[l_{13}-l_{11}-1\right]\right\} \times
\end{aligned}
$$




$$
\begin{aligned}
& \times \frac{1}{[2 l+2]}\left(\frac{\left[l_{13}-l_{11}\right]}{\left[l_{11}-l_{23}\right]}\right)^{1 / 2}(m)_{6}^{+31} \\
& -\frac{1}{a_{7}}\left(\frac{p}{q}\right)^{2(l-s)+l^{\prime}-s^{\prime}-4} \frac{\left([2 l][2 l-1]\left[l_{13}-l_{11}-1\right]\left[l_{13}-l_{11}\right]\right)^{1 / 2}}{[2][2 l+1][2 l+2]}(m)_{7}^{+31}, \\
& |1,0,1,0 ;(m)\rangle=\frac{1}{a_{8}} q^{-1} p^{-2\left(l^{\prime}+s^{\prime}\right)}\left(\frac{q}{p}\right)^{-l+s}\left(\frac{\left[l_{33}-l_{31}+1\right]\left[l_{33}-l_{31}+2\right]}{\left[2 l^{\prime}+1\right]\left[2 l^{\prime}+2\right]}\right)^{1 / 2}(m)_{8}^{-11} \\
& +\frac{1}{a_{9}} q^{-1} p^{2\left(l^{\prime}-s^{\prime}\right)-1}\left(\frac{p}{q}\right)^{l-s} \frac{\left(\left[l_{33}-l_{31}+1\right]\left[l_{31}-l_{43}-1\right]\right)^{1 / 2}}{\left[2 l^{\prime}+2\right]}(m)_{9}^{-11} \\
& -\frac{1}{a_{10}}\left(\frac{p}{q}\right)^{l-s} \frac{q p^{2\left(l^{\prime}-s^{\prime}\right)-1}}{[2]\left[2 l^{\prime}+1\right]\left[2 l^{\prime}+2\right]} \times \\
& \times\left(\left[2 l^{\prime}\right]\left[2 l^{\prime}-1\right]\left[l_{31}-l_{43}-2\right]\left[l_{31}-l_{43}-1\right]\right)^{1 / 2}(m)_{10}^{-11}, \\
& E^{(+2)} \equiv|0,1,1,0 ;(m)\rangle+q^{-1}|1,0,0,1 ;(m)\rangle \\
& =\frac{1}{a_{8}} q^{-1} p^{-l^{\prime}-s^{\prime}-1}\left(\frac{p}{q}\right)^{l-s+1}[2]\left(\frac{\left[l_{33}-l_{31}+1\right]\left[l_{31}-l_{43}\right]}{\left[2 l^{\prime}+1\right]\left[2 l^{\prime}+2\right]}\right)^{1 / 2}(m)_{8}^{-11+31} \\
& +\frac{1}{a_{9}}\left(\frac{p}{q}\right)^{l-s+l^{\prime}-s^{\prime}-2} \frac{p^{l^{\prime}-s^{\prime}}}{q\left[2 l^{\prime}+2\right]} \times \\
& \times\left\{[2][2 l-1]\left[l_{33}-l_{31}\right]-\left[2 l^{\prime}\right]\left(q^{-2}\left[l_{33}-l_{31}\right]+p\left[l_{33}-l_{31}-1\right]\right)\right\}(m)_{9}^{-11+31} \\
& +\frac{1}{a_{10}}\left(\frac{p}{q}\right)^{l-s+l^{\prime}-s^{\prime}-2} \frac{p^{l^{\prime}-s^{\prime}}}{q\left[2 l^{\prime}+1\right]\left[2 l^{\prime}+2\right]} \times \\
& \times\left([2 l][2 l-1]\left[l_{33}-l_{31}\right]\left[l_{31}-l_{43}-1\right]\right)^{1 / 2}(m)_{10}^{-11+31}, \\
& |0,1,0,1 ;(m)\rangle=\frac{1}{a_{8}}\left(\frac{p}{q}\right)^{l-s}\left(\frac{\left[l_{31}-l_{43}\right]\left[l_{31}-l_{43}+1\right]}{\left[2 l^{\prime}+1\right]\left[2 l^{\prime}+2\right]}\right)^{1 / 2}(m)_{8}^{-11+31+31} \\
& +\frac{1}{a_{9}}\left(\frac{p}{q}\right)^{l-s+2\left(l^{\prime}-s^{\prime}\right)-4} \frac{1}{\left[2 l^{\prime}+2\right]}\left(\frac{\left[l_{33}-l_{31}\right]}{\left[2 l^{\prime}+1\right]}\right)^{1 / 2} \times \\
& \times\left(\frac{p}{q}\left[2 l^{\prime}\right]\left[l_{33}-l_{31}-2\right]-\left[2 l^{\prime}-1\right]\left[l_{33}-l_{31}-1\right]\right)(m)_{9}^{-11+31+31} \\
& -\frac{1}{a_{10}}\left(\frac{p}{q}\right)^{l-s+2\left(l^{\prime}-s^{\prime}\right)-4} \frac{1}{[2]\left[2 l^{\prime}+1\right]\left[2 l^{\prime}+2\right]} \times \\
& \times\left(\left[2 l^{\prime}\right]\left[2 l^{\prime}-1\right]\left[l_{33}-l_{31}-1\right]\left[l_{33}-l_{31}\right]\right)^{1 / 2}(m)_{10}^{-11+31+31},
\end{aligned}
$$




$$
\begin{aligned}
& |1,1,1,0 ;(m)\rangle=q^{-l+s-2} p^{l^{\prime}-s^{\prime}-2}\left(\frac{p}{q}\right)^{l-s+l^{\prime}-s^{\prime}} \times \\
& \times\left\{\frac{1}{a_{11}}\left(\frac{\left[l_{13}-l_{11}+1\right]\left[l_{33}-l_{31}+1\right]}{[2 l+1]\left[2 l^{\prime}+1\right]}\right)^{1 / 2}(m)_{11}^{-11-11+31}\right. \\
& +\frac{1}{a_{12}} \frac{q}{[2 l+1]}\left(\frac{[2 l]\left[l_{11}-l_{23}-1\right]\left[l_{33}-l_{31}+1\right]}{\left[2 l^{\prime}+1\right]}\right)^{1 / 2}(m)_{12}^{-11-11+31} \\
& +\frac{1}{a_{13}} \frac{q^{2}}{\left[2 l^{\prime}+1\right]}\left(\frac{\left[l_{13}-l_{11}+1\right]\left[2 l^{\prime}\right]\left[l_{31}-l_{43}-1\right]}{[2 l+1]}\right)^{1 / 2}(m)_{13}^{-11-11+31} \\
& \left.+\frac{1}{a_{14}} \frac{q^{3}}{[2 l+1]\left[2 l^{\prime}+1\right]}\left([2 l]\left[l_{11}-l_{23}-1\right]\left[2 l^{\prime}\right]\left[l_{31}-l_{43}-1\right]\right)^{1 / 2}(m)_{14}^{-11-11+31}\right\}, \\
& |1,0,1,1 ;(m)\rangle=p^{l^{\prime}-s^{\prime}}\left(\frac{p}{q}\right)^{2(l-s)+l^{\prime}-s^{\prime}-3}\left\{\frac{1}{a_{11}} q^{-3}\left(q[2 l]\left[l_{13}-l_{11}\right]-p[2 l+1]\left[l_{13}-l_{11}-1\right]\right) \times\right. \\
& \times\left(p\left[2 l^{\prime}+1\right]-q^{2}\left[2 l^{\prime}\right]\right)\left(\frac{\left[l_{33}-l_{31}+1\right]}{[2 l+1]\left[2 l^{\prime}+1\right]\left[l_{11}-l_{23}\right]}\right)^{1 / 2}(m)_{11}^{-11+31} \\
& -\frac{1}{a_{12}} \frac{q^{-2}}{[2 l+1]}\left(p\left[2 l^{\prime}+1\right]-q^{2}\left[2 l^{\prime}\right]\right) \times \\
& \times\left(\frac{[2 l]\left[l_{13}-l_{11}\right]\left[l_{33}-l_{31}+1\right]}{\left[2 l^{\prime}+1\right]}\right)^{1 / 2}(m)_{12}^{-11+31} \\
& -\frac{1}{a_{13}} \frac{q^{-1}}{\left[2 l^{\prime}+1\right]}\left(q^{-1}[2 l]\left[l_{13}-l_{11}\right]-p[2 l+1]\left[l_{13}-l_{11}-1\right]\right) \times \\
& \times\left(\frac{\left[2 l^{\prime}\right]\left[l_{31}-l_{43}-1\right]}{[2 l+1]\left[l_{11}-l_{23}\right]}\right)^{1 / 2}(m)_{13}^{-11+31} \\
& \left.+\frac{1}{a_{14}} \frac{1}{[2 l+1]\left[2 l^{\prime}+1\right]}\left([2 l]\left[l_{13}-l_{11}\right]\left[2 l^{\prime}\right]\left[l_{31}-l_{43}-1\right]\right)^{1 / 2}(m)_{14}^{-11+31}\right\}, \\
& |1,1,0,1 ;(m)\rangle=q^{-l+s-3}\left(\frac{p}{q}\right)^{l-s+2\left(l^{\prime}-s^{\prime}\right)-4}\left\{\frac{1}{a_{11}}\left(q[2 l]-p^{2}[2 l+1]\right) \times\right. \\
& \times\left(p\left[2 l^{\prime}+1\right]\left[l_{33}-l_{31}-1\right]-q\left[2 l^{\prime}\right]\left[l_{33}-l_{31}\right]\right) \\
& \times\left(\frac{\left[l_{13}-l_{11}+1\right]}{[2 l+1]\left[2 l^{\prime}+1\right]\left[l_{31}-l_{43}\right]}\right)^{1 / 2}(m)_{11}^{-11-11+31+31} \\
& -\frac{1}{a_{12}} \frac{q}{[2 l+1]}\left(p\left[2 l^{\prime}+1\right]\left[l_{33}-l_{31}-1\right]-q\left[2 l^{\prime}\right]\left[l_{33}-l_{31}\right] \times\right) \\
& \times\left(\frac{[2 l]\left[l_{11}-l_{23}-1\right]}{\left[2 l^{\prime}+1\right]\left[l_{31}-l_{43}\right]}\right)^{1 / 2}(m)_{12}^{-11-11+31+31}
\end{aligned}
$$




$$
\begin{aligned}
& +\frac{1}{a_{13}} \frac{q}{\left[2 l^{\prime}+1\right]}\left(q[2 l]-p^{2}[2 l+1]\right) \times \\
& \times\left(\frac{\left[l_{13}-l_{11}+1\right]\left[2 l^{\prime}\right]\left[l_{33}-l_{31}\right]}{[2 l+1]}\right)^{1 / 2}(m)_{13}^{-11-11+31+31} \\
& \left.+\frac{1}{a_{14}} \frac{q^{2}}{[2 l+1]\left[2 l^{\prime}+1\right]}\left([2 l]\left[l_{11}-l_{23}-1\right]\left[2 l^{\prime}\right]\left[l_{33}-l_{31}\right]\right)^{1 / 2}(m)_{14}^{-11-11+31+31}\right\},
\end{aligned}
$$$$
|0,1,1,1 ;(m)\rangle=\left(\frac{p}{q}\right)^{2\left(l-s+l^{\prime}-s^{\prime}-2\right)}\left\{\frac{1}{a_{11}} q^{-2}\left(p[2 l+1]\left[l_{13}-l_{11}-1\right]-q[2 l]\left[l_{13}-l_{11}\right]\right) \times\right.
$$$$
\times\left(p\left[2 l^{\prime}+1\right]\left[l_{33}-l_{31}-1\right]-q\left[2 l^{\prime}\right]\left[l_{33}-l_{31}\right]\right) \times
$$$$
\times\left(\frac{1}{[2 l+1]\left[2 l^{\prime}+1\right]\left[l_{11}-l_{23}\right]\left[l_{31}-l_{43}\right]}\right)^{1 / 2}(m)_{11}^{-11+31+31}
$$$$
+\frac{1}{a_{12}} \frac{q^{-1}}{[2 l+1]}\left(p\left[2 l^{\prime}+1\right]\left[l_{33}-l_{31}-1\right]-q\left[2 l^{\prime}\right]\left[l_{33}-l_{31}\right]\right) \times
$$$$
\times\left(\frac{[2 l]\left[l_{13}-l_{11}\right]}{\left[2 l^{\prime}+1\right]\left[l_{31}-l_{43}\right]}\right)^{1 / 2}(m)_{12}^{-11+31+31}
$$$$
+\frac{1}{a_{13}} \frac{q^{-1}}{\left[2 l^{\prime}+1\right]}\left(p[2 l+1]\left[l_{13}-l_{11}-1\right]-q[2 l]\left[l_{13}-l_{11}\right]\right) \times
$$$$
\times\left(\frac{\left[2 l^{\prime}\right]\left[l_{33}-l_{31}\right]}{[2 l+1]\left[l_{11}-l_{23}\right]}\right)^{1 / 2}(m)_{13}^{-11+31+31}
$$$$
\left.-\frac{1}{a_{14}} \frac{1}{[2 l+1]\left[2 l^{\prime}+1\right]}\left([2 l]\left[l_{13}-l_{11}\right]\left[2 l^{\prime}\right]\left[l_{33}-l_{31}\right]\right)^{1 / 2}(m)_{14}^{-11+31+31}\right\},
$$

$|1,1,1,1 ;(m)\rangle=\frac{1}{a_{15}}(m)^{-11-11+31+31}$. 